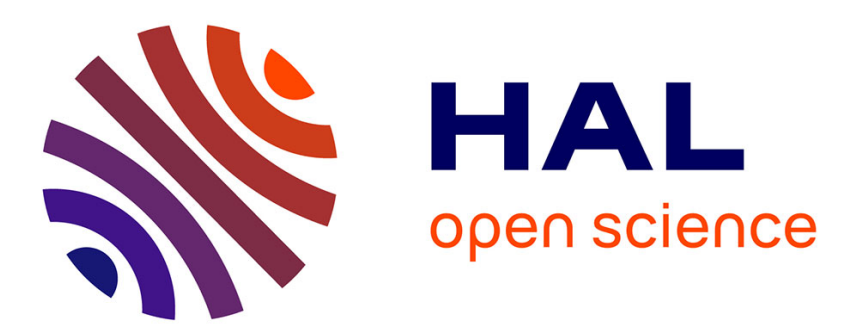

\title{
Higher order interfacial effects for elastic waves in one dimensional phononic crystals via the Lagrange-Hamilton's principle
}

\author{
Frédéric Lebon, Raffaella Rizzoni
}

\section{- To cite this version:}

Frédéric Lebon, Raffaella Rizzoni. Higher order interfacial effects for elastic waves in one dimensional phononic crystals via the Lagrange-Hamilton's principle. European Journal of Mechanics - A/Solids, 2018, 10.1016/j.euromechsol.2017.08.014 . hal-02021009

\section{HAL Id: hal-02021009 \\ https://hal.science/hal-02021009}

Submitted on 11 Jun 2019

HAL is a multi-disciplinary open access archive for the deposit and dissemination of scientific research documents, whether they are published or not. The documents may come from teaching and research institutions in France or abroad, or from public or private research centers.
L'archive ouverte pluridisciplinaire HAL, est destinée au dépôt et à la diffusion de documents scientifiques de niveau recherche, publiés ou non, émanant des établissements d'enseignement et de recherche français ou étrangers, des laboratoires publics ou privés. 


\title{
Higher order interfacial effects for elastic waves in one dimensional phononic crystals via the Lagrange-Hamilton's principle
}

\author{
F. Lebon ${ }^{\text {a }}$, R. Rizzoni ${ }^{\text {b, * }}$ \\ a Aix-Marseille University, CNRS, Centrale Marseille, LMA, Marseille, France \\ ${ }^{\mathrm{b}}$ Department of Engineering, University of Ferrara, Italy
}

\begin{abstract}
This work proposes new transmission conditions at the interfaces between the layers of a threedimensional composite structures. The proposed transmission conditions are obtained by applying the asymptotic expansion technique in the framework of Lagrange-Hamilton's principle. The proposed conditions take into account interfacial effects of higher order, thus representing an extension of the classical zero-thickness interface models. In particular, the (small) thickness of the interface together with its inertia, stiffness and anisotropy are accounted for. The effect of the transmission conditions on the band structure of Bloch-Floquet waves propagating in a one dimensional phononic crystal is discussed based on numerical results.
\end{abstract}

\section{Introduction}

Phononic crystals are composites with a periodic structure made of materials with different elastic constants and densities. In these composites, elastic waves with frequencies within a specific range (the phononic bandgap) are not allowed to propagate. Therefore, phononic crystals present innovative filtering properties and offer possibilities for controlling sound and heat propagation (Kushwaha et al., 1993; Jensen, 2003; Ghazaryan and Piliposyan, 2011; Maldovan, 2013).

Many authors have studied the effect of material properties on phononic band gaps (see (Vasseur et al., 2001; Wu et al., 2004; Maldovan, 2013; Chen et al., 2014) and references therein) and found that microstructured materials are able to control sound, whereas to control heat, nanostructures are generally required. For a fine-scaled material with a large ratio of interfacial region to the bulk, the influence of surface characteristics can be substantial and it is thus fundamental to propose reliable and efficient models able to account for interfacial effects.

In the literature, a very large number of interface model have been developed (see, for example (Challamel and Girhammar, 2011;

\footnotetext{
* Corresponding author.

E-mail addresses: lebon@lma.cnrs-mrs.fr (F. Lebon), raffaella.rizzoni@unife.it (R. Rizzoni).
}

Benveniste and Miloh, 2001; Hashin, 2002; Klarbring, 1991; Bövik 1994; Lebon and Zaittouni, 2010; Nairn, 2007; Benveniste, 2013; Li et al., 2015)). We can classify these models into two large families: phenomenological models, essentially built from experimental data, and deductive models based on micro-mechanical analyses. In the present paper, we deal with the second family.

The application of asymptotic techniques to obtain models of interfaces is now well established (Benveniste, 2006; Krasuki and Lenci, 2000; Lebon et al., 1997; Rizzoni and Lebon, 2012, 2013; Rizzoni et al., 2014; Serpilli, 2015; Serpilli and Lenci, 2016). The idea behind this application is the replacement of a thin, elastic, anisotropic interphase by a proper interface model; the equivalence between the two models is established by studying the asymptotic behavior of the interphase as its thickness becomes smaller and smaller. In Section 2, the problem of a composite made of three deformable solids (two adherents and a thin interphase) perfectly bonded together is introduced in the framework of elastodynamics. The Lagrangian problem is introduced and expanded with respect to the small parameter (the interphase thickness). In Section 3, four sub-problems are studied, allowing us to derive the interfacial displacement and traction jump relations at each level of expansion. In particular, higher order levels of the expansion are taken into account.

In Section 4, the jump relations are reformulated into a general elastic imperfect interface model in such a way that they take 
simpler and compact but equivalent forms, particularly convenient for later use. It is also shown that the present formulation unifies and extends four widely used interface models, the perfect interface, the mass interface, the spring interface and the spring-mass interface, thus representing an enrichment of the classical interface models due to high order interfacial effects. Notably, the presence of first order derivates of the displacement and stress vector fields make the proposed imperfect interface model nonlocal in character.

In Section 5, the imperfect interface model is applied to estimate the interfacial effects of the periodic structure on bandgaps of a one-dimensional phononic crystal with imperfect contacts between the two constituent layers. The standard transfer matrix approach is employed (Lekner, 1994; Rokhlin and Wana, 1991; Rokhlin and Wang, 1992). In particular, an additional interlayer matrix is introduced, taking into account the imperfect contact. The dispersion equation is solved numerically and the dispersion curves are shown in the Brillouin zone. The band gaps of the phononic crystal with imperfect contact are compared with those obtained with perfect contact. In particular, the effects of the small thickness of the imperfect interface, of its inertia and stiffness on the band structure of the laminated phononic crystal are discussed on the basis of the numerical results.

\section{Statement of the problem}

In the following a composite body made of three deformable solids, two elastic adherents and a thin elastic adhesive, is considered (cf. Fig. 1). At the initial time $t_{1}$, the composite occupies the bounded domain $\Omega^{\varepsilon}$ depending on a small parameter $\varepsilon$ which is the constant thickness of the adhesive. An orthonormal Cartesian basis $\left(O, \mathbf{e}_{1}, \mathbf{e}_{2}, \mathbf{e}_{3}\right)$ is introduced and $\mathbf{x}=\left(x_{1}, x_{2}, x_{3}\right)$ is taken to denote the position of a particle. The adhesive occupies the initial domain $B^{\varepsilon}$, defined by $B^{\varepsilon}=\left\{\left(x_{1}, x_{2}, x_{3}\right) \in \Omega^{\varepsilon}:\left|x_{3}\right|<\frac{\varepsilon}{2}\right\}$. We take $\partial B^{\varepsilon}$ to denote the boundary of $B^{\varepsilon}$, which is supposed to be sufficiently smooth. Thus, the origin of the Cartesian basis lies at the center of the adhesive midplane and the $x_{3}$ - axis runs perpendicular to the open bounded set $S=\left\{\left(x_{1}, x_{2}, x_{3}\right) \in \Omega^{\varepsilon}: x_{3}=0\right\}$, which in the following will be called the interface. The adherents occupy respectively the initial domains $\Omega_{ \pm}^{\varepsilon}$ defined by $\Omega_{ \pm}^{\varepsilon}=\left\{\left(x_{1}, x_{2}, x_{3}\right) \in \Omega: \pm x_{3}>\frac{\varepsilon}{2}\right\}$. We take $\partial \Omega_{ \pm}^{\varepsilon}$ to denote the boundary of $\Omega_{ \pm}^{\varepsilon}$, which is supposed to be sufficiently smooth. The two-dimensional domains $S_{ \pm}^{\varepsilon}$ are taken to denote the interfaces between the adhesive and the adherents, $S_{ \pm}^{\varepsilon}=\left\{\left(x_{1}, x_{2}, x_{3}\right) \in \Omega: x_{3}= \pm \frac{\varepsilon}{2}\right\}$. On a part $S_{g}^{ \pm}$of the boundary

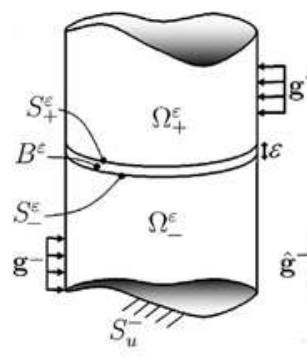

(a)

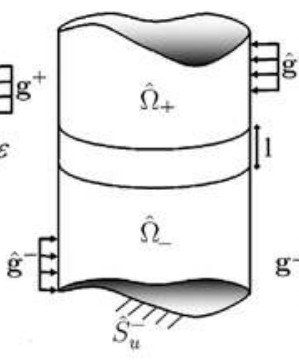

(b)

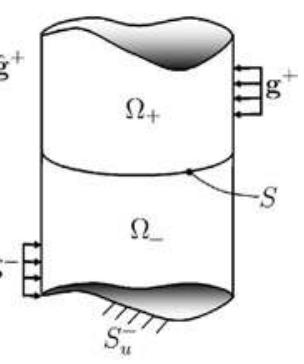

(c)
Fig. 1. Geometry of the composite. Initial reference configuration made of two adherents in perfect contact with a thin adhesive (a); corresponding rescaled configuration with an adhesive of unit thickness (b); limit configuration obtained as the thickness $\varepsilon$ of the adhesive goes to zero (c). $\partial \Omega^{\varepsilon} / S_{ \pm}^{\varepsilon}$, an external time-dependent load $\mathbf{g}^{ \pm}(t, \mathbf{x}), t \in\left(t_{1}, t_{2}\right)$, is applied, and on a part $S_{u}^{ \pm}$of $\partial \Omega^{\varepsilon} / S_{ \pm}^{\varepsilon}$ such that $S_{g}^{ \pm} \cap S_{u}^{ \pm}=\emptyset$, the displacement is imposed to vanish. Moreover, it is assumed that $S_{u}^{ \pm} \cap B^{\varepsilon}=\emptyset, S_{g}^{ \pm} \cap B^{\varepsilon}=\emptyset$ and $S_{u}^{ \pm} \cup S_{g}^{ \pm} \cup S_{ \pm}^{\varepsilon}=\partial \Omega_{ \pm}^{\varepsilon}$. We take $S_{l}^{\varepsilon}$ to denote $\partial B^{\varepsilon} / S_{ \pm}^{\varepsilon}$. The part of the boundary $S_{l}^{\varepsilon}$ is force free. A time-dependent body force $\mathbf{f}^{ \pm}(t, \mathbf{x}), t \in\left(t_{1}, t_{2}\right)$, is applied in $\Omega_{ \pm}^{\varepsilon}$. Let $\mathbf{g}^{ \pm}$and $\mathbf{f}^{ \pm}$be assumed regular functions on $\left[t_{1}, t_{2}\right] \times S_{g}$ and $\left[t_{1}, t_{2}\right] \times \Omega_{ \pm}^{\varepsilon}$, respectively. In the following, $\mathbf{u}^{\varepsilon}(t, \mathbf{x})$ is taken to denote the displacement field, $\boldsymbol{\sigma}^{\varepsilon}(t, \mathbf{x})$ the Cauchy stress tensor and $\mathbf{e}\left(\mathbf{u}^{\varepsilon}\right)$ the strain tensor. Under the small strain hypothesis we have $e_{i j}\left(\mathbf{u}^{\varepsilon}\right)=\frac{1}{2}\left(u_{i, j}^{\varepsilon}+u_{j, i}^{\varepsilon}\right)$, where the comma is the partial derivative.

The two adherents and the adhesive are supposed to be elastic, thus

$\boldsymbol{\sigma}^{\varepsilon}=\mathbf{a}_{ \pm} \mathbf{e}\left(\mathbf{u}^{\varepsilon}\right)$ in $\Omega_{ \pm}^{\varepsilon}$

$\boldsymbol{\sigma}^{\varepsilon}=\mathbf{b}^{\varepsilon} \mathbf{e}\left(\mathbf{u}^{\varepsilon}\right)$ in $B^{\varepsilon}$.

The elasticity tensors $\mathbf{a}_{ \pm}$and $\mathbf{b}^{\varepsilon}$ have the usual properties of symmetry, $S_{i j h k}=S_{h k i j}=S_{j i k h}$, and of positivity, i.e. there exists $\alpha>0$ such that

$S_{i j h k} e_{i j} e_{h k}>\alpha e_{i j} e_{i j}, \quad e_{i j}=e_{j i}$.

We take $\rho^{ \pm}$and $\zeta^{\varepsilon}$ to denote the strictly positive volumetric mass densities in the adherents and in the adhesive, respectively, and []] to denote the jump along $S_{ \pm}^{\varepsilon}$. The equations governing the motion of the composite structure are written as follows:

$\left\{\begin{array}{cc}\sigma_{i j, j}^{\varepsilon}+f_{i}^{ \pm}=\rho_{ \pm} \ddot{u}_{i}^{\varepsilon} & \text { in }\left[t_{1}, t_{2}\right] \times \Omega_{ \pm}^{\varepsilon}, \\ \sigma_{i j}^{\varepsilon} n_{j}=g_{i}^{ \pm} & \text {on }\left[t_{1}, t_{2}\right] \times S_{g}^{ \pm}, \\ \sigma_{i j . j}^{\varepsilon}=\zeta^{\varepsilon} \ddot{u}^{\varepsilon} & \text { in }\left[t_{1}, t_{2}\right] \times B^{\varepsilon}, \\ {\left[\left[u_{i}^{\varepsilon}\right]\right]=0} & \text { on }\left[t_{1}, t_{2}\right] \times S_{ \pm}^{\varepsilon}, \\ u_{i}^{\varepsilon}=0 & \text { on }\left[t_{1}, t_{2}\right] \times S_{u}^{ \pm}, \\ \sigma_{i j}^{\varepsilon}=a_{i j h k}^{ \pm} e_{h k}\left(u^{\varepsilon}\right) & \text { in }\left[t_{1}, t_{2}\right] \times \Omega_{ \pm}^{\varepsilon}, \\ \sigma_{i j}^{\varepsilon}=b_{i j h k}^{\varepsilon} e_{h k}\left(u^{\varepsilon}\right) & \text { in }\left[t_{1}, t_{2}\right] \times B^{\varepsilon}, \\ u_{i}^{\varepsilon}=U_{i}^{\varepsilon}, & \text { for } t=t_{1}, \text { in } \Omega^{\varepsilon}, \\ \dot{u}_{i}^{\varepsilon}=v_{i}^{\varepsilon}, & \text { for } t=t_{1}, \text { in } \Omega^{\varepsilon},\end{array}\right.$

where $\dot{u}_{i}$ and $\ddot{u}_{i}$ are the first and second derivatives in time of $u_{i}$, respectively, and $U_{i}$ (resp. $v_{i}$ ) are the initial displacement (resp. velocity) data. It is remarked here, that (4) implies that $\left[\left[\sigma_{i}^{\varepsilon}\right]\right]=0$ on $\left[t_{1}, t_{2}\right] \times S_{ \pm}^{\varepsilon}$. Note that $b_{i j h k}^{\varepsilon}$ and $\zeta^{\varepsilon}$ can depend on $\varepsilon$. If $f^{ \pm} \in L^{2}\left(\left[t_{1}, t_{2}\right] ; H^{1}\left(\Omega^{\varepsilon}, R^{3}\right)\right)$ and $g^{ \pm} \in L^{2}\left(\left[t_{1}, t_{2}\right] ; H^{1}\left(S_{g}^{ \pm}, R^{3}\right)\right)$, then problem (4) has an unique solution in $H^{1}\left(\left[t_{1}, t_{2}\right] ; H^{1}\left(\Omega^{\varepsilon}, R^{3}\right)\right)$ (Ciarlet, 1976; Lions and Magenes, 1968). In the following, we take \|\| to denote the usual euclidian norm in $R^{3}$. The Lagrangian is introduced

$\mathscr{L}\left(\mathbf{u}^{\varepsilon}\right)=T^{\varepsilon}\left(\dot{\mathbf{u}}^{\varepsilon}\right)-E^{\varepsilon}\left(\mathbf{u}^{\varepsilon}\right)$,

where $T^{\varepsilon}$ is the total kinetic energy, sum of the kinetic energies of the adherents and the adhesive,

$$
\begin{gathered}
T^{\varepsilon}\left(\dot{\mathbf{u}}^{\varepsilon}\right)=T_{+}^{\varepsilon}\left(\dot{\mathbf{u}}^{\varepsilon}\right)+T_{-}^{\varepsilon}\left(\dot{\mathbf{u}}^{\varepsilon}\right)+T_{B}^{\varepsilon}\left(\dot{\mathbf{u}}^{\varepsilon}\right), \\
T_{ \pm}^{\varepsilon}\left(\dot{\mathbf{u}}^{\varepsilon}\right)=\frac{1}{2} \int_{\Omega_{ \pm}^{\varepsilon}} \rho_{ \pm}\left\|\dot{\mathbf{u}}^{\varepsilon}\right\|^{2} d x \\
T_{B}^{\varepsilon}\left(\dot{\mathbf{u}}^{\varepsilon}\right)=\frac{1}{2} \int_{B^{\varepsilon}} \zeta^{\varepsilon}\left\|\dot{\mathbf{u}}^{\varepsilon}\right\|^{2} d x
\end{gathered}
$$

and $E^{\varepsilon}$ is the total potential energy 


$$
\begin{gathered}
E^{\varepsilon}\left(\mathbf{u}^{\varepsilon}\right)=E_{+}^{\varepsilon}\left(\mathbf{u}^{\varepsilon}\right)+E_{-}^{\varepsilon}\left(\mathbf{u}^{\varepsilon}\right)+E_{B}^{\varepsilon}\left(\mathbf{u}^{\varepsilon}\right), \\
E_{ \pm}^{\varepsilon}\left(\mathbf{u}^{\varepsilon}\right)=\frac{1}{2} \int_{\Omega_{ \pm}^{\varepsilon}} \mathbf{a}_{ \pm}\left(\mathbf{e}\left(\mathbf{u}^{\varepsilon}\right)\right) \cdot \mathbf{e}\left(\mathbf{u}^{\varepsilon}\right) d x-\int_{\Omega_{ \pm}} \mathbf{f}^{ \pm} \cdot \mathbf{u}^{\varepsilon} d x-\int_{S_{\bar{g}}^{ \pm}} \mathbf{g}^{ \pm} \cdot \mathbf{u}^{\varepsilon} d s_{X}, \\
E_{B}^{\varepsilon}\left(\mathbf{u}^{\varepsilon}\right)=\frac{1}{2} \int_{B^{\varepsilon}} \mathbf{b}^{\varepsilon}\left(\mathbf{e}\left(\mathbf{u}^{\varepsilon}\right)\right) \cdot \mathbf{e}\left(\mathbf{u}^{\varepsilon}\right) d x .
\end{gathered}
$$

The Lagrange-Hamilton's principle states that among admissible motions, the actual motion of the material is such that

$\delta \int_{t_{1}}^{t_{2}} \mathscr{L}^{\varepsilon} d t=0$.

Under suitable regularity assumptions on the domains $\Omega_{+}^{\varepsilon}, B^{\varepsilon}, S$ and on the fields $\mathbf{f}^{ \pm}, \mathbf{g}^{ \pm}, \mathbf{v}^{\varepsilon}, \mathbf{u}^{\varepsilon}$, the Lagrange-Hamilton's principle is equivalent to the equation of motions (4) (Bedford, 1985; Seliger and Whitham, 1968).

\section{A general asymptotic approach}

Since the thickness of the interphase is very small, it is natural to seek the solution of problem $(4,8)$ using asymptotic expansions with respect to the parameter $\varepsilon$. In particular, the following asymptotic series are assumed:

$\left\{\begin{array}{c}\mathbf{u}^{\varepsilon}=\mathbf{u}^{0}+\varepsilon \mathbf{u}^{1}+\varepsilon^{2} \mathbf{u}^{2}+\varepsilon^{3} \mathbf{u}^{3}+o\left(\varepsilon^{3}\right) \\ \boldsymbol{\sigma}^{\varepsilon}=\boldsymbol{\sigma}^{0}+\varepsilon \boldsymbol{\sigma}^{1}+\varepsilon^{2} \boldsymbol{\sigma}^{2}+\varepsilon^{3} \boldsymbol{\sigma}^{3}+o\left(\varepsilon^{3}\right) .\end{array}\right.$

The domain is then rescaled using a classical change of variable (Ciarlet, 1997; Lebon and Rizzoni, 2011):

$\left(z_{1}, z_{2}, z_{3}\right)=\overline{\mathbf{p}}\left(x_{1}, x_{2}, x_{3}\right):=\left(x_{1}, x_{2}, x_{3} \varepsilon^{-1}\right),\left(x_{1}, x_{2}, x_{3}\right) \in B^{\varepsilon}$,

$\left(z_{1}, z_{2}, z_{3}\right)=\widehat{\mathbf{p}}\left(x_{1}, x_{2}, x_{3}\right):=\left(x_{1}, x_{2}, x_{3} \pm \frac{\varepsilon}{2} \mp \frac{1}{2}\right),\left(x_{1}, x_{2}, x_{3}\right) \in \Omega_{ \pm}^{\varepsilon}$,

and let $\bar{B}$ be the rescaled interphase

$\bar{B}=\left\{\left(z_{1}, z_{2}, z_{3}\right) \in R^{3}:\left(z_{1}, z_{2}\right) \in S,\left|z_{3}\right|<\frac{1}{2}\right\}$,

and $\widehat{\Omega}_{ \pm}$the rescaled adherents. The displacement fields from the rescaled adherents and from the rescaled interphase are

$\widehat{\mathbf{u}}_{ \pm}^{\varepsilon}\left(z_{1}, z_{2}, z_{3}, t\right):=\left(\mathbf{u}^{\varepsilon} \circ \widehat{\mathbf{p}}^{-1}\right)\left(z_{1}, z_{2}, z_{3}\right), \quad\left(z_{1}, z_{2}, z_{3}\right) \in \widehat{\Omega}_{ \pm}$,

$\overline{\mathbf{u}}^{\varepsilon}\left(z_{1}, z_{2}, z_{3}, t\right):=\left(\mathbf{u}^{\varepsilon} \circ \overline{\mathbf{p}}^{-1}\right)\left(z_{1}, z_{2}, z_{3}\right), \quad\left(z_{1}, z_{2}, z_{3}\right) \in \bar{B}$,

respectively. The conditions of perfect adherence at the interfaces $S_{ \pm}^{\varepsilon}$ can be written as

$\widehat{\mathbf{u}}_{ \pm}^{\varepsilon}\left(z_{1}, z_{2}, \pm \frac{1}{2}, t\right)=\overline{\mathbf{u}}^{\varepsilon}\left(z_{1}, z_{2}, \pm \frac{1}{2}, t\right), \quad\left(z_{1}, z_{2}\right) \in S$, $\left.\mathbf{u}^{\varepsilon}\left(x_{1}, x_{2},\left( \pm \frac{\varepsilon}{2}\right)^{\mp}, t\right)=\widehat{\mathbf{u}}_{ \pm}^{\varepsilon} z_{1}, z_{2},\left( \pm \frac{1}{2}\right)^{\mp}, t\right),\left(x_{1}, x_{2}\right),\left(z_{1}, z_{2}, t\right) \in S$

$\mathbf{u}^{\varepsilon}\left(x_{1}, x_{2},\left( \pm \frac{\varepsilon}{2}\right)^{ \pm}, t\right)=\overline{\mathbf{u}}^{\varepsilon}\left(z_{1}, z_{2},\left( \pm \frac{1}{2}\right)^{ \pm}, t\right),\left(x_{1}, x_{2}\right),\left(z_{1}, z_{2}\right) \in S$.

Using these notations, the kinetic and potential energies can be re-written as

$$
\begin{array}{lll}
\mathscr{T}^{\varepsilon}\left(\dot{\hat{\mathbf{u}}}_{ \pm}^{\varepsilon}, \dot{\overline{\mathbf{u}}}^{\varepsilon}\right) & =\mathscr{T}_{+}^{\varepsilon}\left(\dot{\hat{\mathbf{u}}}_{+}^{\varepsilon}\right)+\mathscr{T}_{-}^{\varepsilon}\left(\dot{\hat{\mathbf{u}}}_{-}^{\varepsilon}\right)+\mathscr{T}_{B}^{\varepsilon}\left(\dot{\overline{\mathbf{u}}}^{\varepsilon}\right), \\
\mathscr{T}_{ \pm}^{\varepsilon}\left(\dot{\hat{\mathbf{u}}}_{ \pm}^{\varepsilon}\right) & = & \frac{1}{2} \int_{\widehat{\Omega}_{ \pm}} \rho_{ \pm}\left\|\dot{\hat{\mathbf{u}}}_{ \pm}^{\varepsilon}\right\|^{2} d z, \\
\mathscr{T}_{B}^{\varepsilon}\left(\dot{\overline{\mathbf{u}}}^{\varepsilon}\right) & = & \frac{1}{2} \int_{\bar{B}} \varepsilon \zeta^{\varepsilon}\left\|\dot{\mathbf{u}}^{\varepsilon}\right\|^{2} d z,
\end{array}
$$

$$
\begin{array}{cc}
\mathscr{E}^{\varepsilon}\left(\widehat{\mathbf{u}}_{ \pm}^{\varepsilon}, \overline{\mathbf{u}}^{\varepsilon}\right)= & \mathscr{E}_{+}^{\varepsilon}\left(\widehat{\mathbf{u}}_{+}^{\varepsilon}\right)+\mathscr{E}_{-}^{\varepsilon}\left(\widehat{\mathbf{u}}_{-}^{\varepsilon}\right)+\mathscr{E}_{B}^{\varepsilon}\left(\overline{\mathbf{u}}^{\varepsilon}\right), \\
\mathscr{E}_{ \pm}^{\varepsilon}\left(\widehat{\mathbf{u}}_{ \pm}^{\varepsilon}\right)=\frac{1}{2} \int_{\widehat{\Omega}_{ \pm}} \mathbf{a}_{ \pm}\left(\mathbf{e}\left(\widehat{\mathbf{u}}_{ \pm}^{\varepsilon}\right)\right) \cdot \mathbf{e}\left(\widehat{\mathbf{u}}_{ \pm}^{\varepsilon}\right) d z-\int_{\widehat{\Omega}_{ \pm}} \widehat{\mathbf{f}}^{ \pm} \cdot \widehat{\mathbf{u}}_{ \pm}^{\varepsilon} d z-\int_{\widehat{S}_{g}} \widehat{\mathbf{g}}^{ \pm} \cdot \widehat{\mathbf{u}}_{ \pm}^{\varepsilon} d s_{z}, \\
\mathscr{E}_{B}^{\varepsilon}\left(\overline{\mathbf{u}}^{\varepsilon}\right)= & \frac{1}{2} \int_{\bar{B}} \varepsilon \mathbf{b}^{\varepsilon}\left(\mathbf{e}\left(\overline{\mathbf{u}}^{\varepsilon}\right)\right) \cdot \mathbf{e}\left(\overline{\mathbf{u}}^{\varepsilon}\right) d z,
\end{array}
$$

where $\widehat{\mathbf{f}}^{ \pm}$and $\widehat{\mathbf{g}}^{ \pm}$are the rescaled body and surface forces. We assume that

$\zeta^{\varepsilon}=\zeta^{0}+\varepsilon \zeta^{1}+o(\varepsilon)$

$\mathbf{K}^{\varepsilon, j l}=\mathbf{K}_{0}^{j l}+\varepsilon \mathbf{K}_{1}^{j l}+o(\varepsilon)$,

where

$K_{k i}^{\varepsilon, j l}:=b_{i j k l}^{\varepsilon}$.

The rescaled kinetic and potential energies in the adhesive then become

$$
\begin{aligned}
& \mathscr{T}_{B}^{\varepsilon}\left(\dot{\mathbf{u}}^{\varepsilon}\right) \approx \int_{\bar{B}} \frac{1}{2}\left(\varepsilon \zeta^{0}+\varepsilon^{2} \zeta^{1}\right)\left(\dot{\mathbf{u}}^{\varepsilon}\right)^{2} d z, \\
& \mathscr{E}_{B}^{\varepsilon}\left(\overline{\mathbf{u}}^{\varepsilon}\right) \approx \int_{\bar{B}} \frac{1}{2}\left(\varepsilon^{-1} \mathbf{K}_{0}^{33}\left(\overline{\mathbf{u}}_{, 3}^{\varepsilon}\right) \cdot \overline{\mathbf{u}}_{, 3}^{\varepsilon}+\left(\mathbf{K}_{1}^{33}\left(\overline{\mathbf{u}}_{, 3}^{\varepsilon}\right) \cdot \overline{\mathbf{u}}_{, 3}^{\varepsilon}\right.\right. \\
& \left.+2 \mathbf{K}_{0}^{\alpha 3}\left(\overline{\mathbf{u}}_{, \alpha}^{\varepsilon}\right) \cdot \overline{\mathbf{u}}_{, 3}^{\varepsilon}\right)+\varepsilon\left(2 \mathbf{K}_{1}^{\alpha 3}\left(\overline{\mathbf{u}}_{, \alpha}^{\varepsilon}\right) \cdot \overline{\mathbf{u}}_{, 3}^{\varepsilon}+\mathbf{K}_{0}^{\alpha \beta}\left(\overline{\mathbf{u}}_{, \alpha}^{\varepsilon}\right) \cdot \overline{\mathbf{u}}_{, \beta}^{\varepsilon}\right) \\
& \left.+\varepsilon^{2} \mathbf{K}_{1}^{\alpha \beta}\left(\overline{\mathbf{u}}_{, \alpha}^{\varepsilon}\right) \cdot \overline{\mathbf{u}}_{, \beta}^{\varepsilon}\right) d z,
\end{aligned}
$$

respectively, and correspondingly the Lagrangian becomes

$\breve{\mathscr{L}}^{\varepsilon}\left(\widehat{\mathbf{u}}_{ \pm}^{\varepsilon}, \overline{\mathbf{u}}^{\varepsilon}, \dot{\hat{u}}_{ \pm}^{\varepsilon}, \dot{\bar{u}}^{\varepsilon}\right)=\mathscr{T}^{\varepsilon}\left(\dot{\hat{u}}_{ \pm}^{\varepsilon}, \dot{\bar{u}}^{\varepsilon}\right)-\mathscr{E}^{\varepsilon}\left(\widehat{\mathbf{u}}_{ \pm}^{\varepsilon}, \overline{\mathbf{u}}^{\varepsilon}\right)$.

The Lagrange-Hamilton's principle is now written as 
$\delta \int_{t_{1}}^{t_{2}} \breve{\mathscr{L}}^{\varepsilon} d t=0$

Substituting the change of variables into the expression of the displacement and stress fields the following asymptotic expansions are deduced

$\left\{\begin{array}{l}\widehat{\boldsymbol{\sigma}}^{\varepsilon}=\widehat{\boldsymbol{\sigma}}^{0}+\varepsilon \widehat{\boldsymbol{\sigma}}^{1}+\varepsilon^{2} \widehat{\boldsymbol{\sigma}}^{2}+\varepsilon^{3} \widehat{\boldsymbol{\sigma}}^{3}+o\left(\varepsilon^{3}\right) \\ \widehat{\mathbf{u}}_{ \pm}^{\varepsilon}=\widehat{\mathbf{u}}_{ \pm}^{0}+\varepsilon \widehat{\mathbf{u}}_{ \pm}^{1}+\varepsilon^{2} \widehat{\mathbf{u}}_{ \pm}^{2}+\varepsilon^{3} \widehat{\mathbf{u}}_{ \pm}^{3}+o\left(\varepsilon^{3}\right) \\ \overline{\boldsymbol{\sigma}}^{\varepsilon}=\overline{\boldsymbol{\sigma}}^{0}+\varepsilon \overline{\boldsymbol{\sigma}}^{1}+\varepsilon^{2} \overline{\boldsymbol{\sigma}}^{2}+\varepsilon^{3} \overline{\boldsymbol{\sigma}}^{3}+o\left(\varepsilon^{3}\right) \\ \overline{\mathbf{u}}^{\varepsilon}=\overline{\mathbf{u}}^{0}+\varepsilon \overline{\mathbf{u}}^{1}+\varepsilon^{2} \overline{\mathbf{u}}^{2}+\varepsilon^{3} \overline{\mathbf{u}}^{3}+o\left(\varepsilon^{3}\right),\end{array}\right.$

in the rescaled adhesive and adherents, respectively. Accordingly, the kinetic energy takes the form

$$
\begin{aligned}
& \mathscr{T}_{ \pm}^{\varepsilon}\left(\dot{\overrightarrow{\mathbf{u}}}_{ \pm}^{\varepsilon}\right)=\mathscr{T}_{ \pm}^{0}\left(\dot{\overrightarrow{\mathbf{u}}}_{ \pm}^{0}\right)+\varepsilon \mathscr{T}_{ \pm}^{1}\left(\dot{\overrightarrow{\mathbf{u}}}_{ \pm}^{0}, \dot{\hat{\mathbf{u}}}_{ \pm}^{1}\right)+\varepsilon^{2} \mathscr{T}_{ \pm}^{2}\left(\dot{\overrightarrow{\mathbf{u}}}_{ \pm}^{1}\right)+o\left(\varepsilon^{2}\right), \\
& \mathscr{T}_{ \pm}^{0}\left(\dot{\overrightarrow{\mathbf{u}}}_{ \pm}^{0}\right)=\frac{1}{2} \int_{\widehat{\Omega}_{ \pm}} \rho_{ \pm}\left\|\dot{\hat{\mathbf{u}}}_{ \pm}^{0}\right\|^{2} d z \\
& \mathscr{T}_{ \pm}^{1}\left(\dot{\hat{\mathbf{u}}}_{ \pm}^{0}, \dot{\hat{\mathbf{u}}}_{ \pm}^{1}\right)=\int_{\widehat{\Omega}_{ \pm}} \rho_{ \pm}\left(\dot{\hat{\mathbf{u}}}_{ \pm}^{0} \cdot \dot{\hat{\mathbf{u}}}_{ \pm}^{1}\right) d z \\
& \mathscr{T}_{ \pm}^{2}\left(\dot{\overrightarrow{\mathbf{u}}}_{ \pm}^{0}, \dot{\overrightarrow{\mathbf{u}}}_{ \pm}^{1}, \dot{\widehat{\mathbf{u}}}_{ \pm}^{2}\right)=\frac{1}{2} \int_{\widehat{\Omega}_{ \pm}} \rho_{ \pm}\left(\left\|\dot{\overrightarrow{\mathbf{u}}}_{ \pm}^{1}\right\|^{2}+2 \dot{\overrightarrow{\mathbf{u}}}_{ \pm}^{0} \cdot \dot{\overrightarrow{\mathbf{u}}}_{ \pm}^{2}\right) d z, \\
& \mathscr{T}_{B}^{\varepsilon}\left(\dot{\overline{\mathbf{u}}}^{\varepsilon}\right)=\varepsilon \mathscr{T}_{B}^{1}\left(\dot{\overline{\mathbf{u}}}^{0}\right)+\varepsilon^{2} \mathscr{T}_{B}^{2}\left(\dot{\overline{\mathbf{u}}}^{0}, \dot{\overline{\mathbf{u}}}^{1}\right)+o\left(\varepsilon^{2}\right), \\
& \mathscr{T}_{B}^{1}\left(\dot{\overline{\mathbf{u}}}^{0}\right)=\frac{1}{2} \int_{\bar{B}} \zeta^{0}\left\|\dot{\overline{\mathbf{u}}}^{0}\right\|^{2} d z, \\
& \mathscr{T}_{B}^{2}\left(\dot{\overline{\mathbf{u}}}^{0}, \dot{\overline{\mathbf{u}}}^{1}\right)=\frac{1}{2} \int_{\bar{B}}\left(2 \zeta^{0}\left(\dot{\overline{\mathbf{u}}}^{0} \cdot \dot{\overline{\mathbf{u}}}^{1}\right)+\zeta^{1}\left\|\dot{\mathbf{u}}^{0}\right\|^{2}\right) d z
\end{aligned}
$$

and the potential energy becomes

$$
\begin{aligned}
& \mathscr{E}_{ \pm}^{\varepsilon}\left(\widehat{\mathbf{u}}_{ \pm}^{\varepsilon}\right) \quad=\mathscr{E}_{ \pm}^{0}\left(\widehat{\mathbf{u}}_{ \pm}^{0}\right)+\varepsilon \mathscr{E}_{ \pm}^{1}\left(\widehat{\mathbf{u}}_{ \pm}^{0}, \widehat{\mathbf{u}}_{ \pm}^{1}\right)+\varepsilon^{2} \mathscr{E}_{ \pm}^{2}\left(\widehat{\mathbf{u}}_{ \pm}^{0}, \widehat{\mathbf{u}}_{ \pm}^{1}, \widehat{\mathbf{u}}_{ \pm}^{2}\right)+o\left(\varepsilon^{2}\right), \\
& \mathscr{C}_{ \pm}^{0}\left(\widehat{\mathbf{u}}_{ \pm}^{0}\right)=\frac{1}{2} \int_{\widehat{\Omega}_{ \pm}} \mathbf{a}_{ \pm}\left(\mathbf{e}\left(\widehat{\mathbf{u}}_{ \pm}^{0}\right)\right) \cdot \mathbf{e}\left(\widehat{\mathbf{u}}_{ \pm}^{0}\right) d z-\int_{\widehat{\Omega}_{ \pm}} \widehat{\mathbf{f}}^{ \pm} \cdot \widehat{\mathbf{u}}_{ \pm}^{0} d z-\int_{\widehat{S}_{g}} \widehat{\mathbf{g}}^{ \pm} \cdot \widehat{\mathbf{u}}_{ \pm}^{0} d s_{z}, \\
& \mathscr{E}_{ \pm}^{1}\left(\widehat{\mathbf{u}}_{ \pm}^{0}, \widehat{\mathbf{u}}_{ \pm}^{1}\right)=\int_{\widehat{\Omega}_{ \pm}} \mathbf{a}_{ \pm}\left(\mathbf{e}\left(\widehat{\mathbf{u}}_{ \pm}^{0}\right)\right) \cdot \mathbf{e}\left(\widehat{\mathbf{u}}_{ \pm}^{1}\right) d z-\int_{\widehat{\Omega}_{ \pm}} \widehat{\mathbf{f}}^{ \pm} \cdot \widehat{\mathbf{u}}_{ \pm}^{1} d z-\int_{\widehat{S}_{g}} \widehat{\mathbf{g}}^{ \pm} \cdot \widehat{\mathbf{u}}_{ \pm}^{1} d s_{z}, \\
& \mathscr{E}_{ \pm}^{2}\left(\widehat{\mathbf{u}}_{ \pm}^{0}, \widehat{\mathbf{u}}_{ \pm}^{1}, \widehat{\mathbf{u}}_{ \pm}^{2}\right)=\frac{1}{2} \int_{\widehat{\Omega}_{ \pm}} \mathbf{a}_{ \pm}\left(\mathbf{e}\left(\widehat{\mathbf{u}}_{ \pm}^{1}\right)\right) \cdot \mathbf{e}\left(\widehat{\mathbf{u}}_{ \pm}^{1}\right) d z+\int_{\widehat{\Omega}_{ \pm}} \mathbf{a}_{ \pm}\left(\mathbf{e}\left(\widehat{\mathbf{u}}_{ \pm}^{0}\right)\right) \cdot \mathbf{e}\left(\widehat{\mathbf{u}}_{ \pm}^{2}\right) d z \\
& -\int_{\widehat{\Omega}_{ \pm}} \widehat{\mathbf{f}}^{ \pm} \cdot \widehat{\mathbf{u}}_{ \pm}^{2} d z-\int_{\widehat{S}_{g}} \widehat{\mathbf{g}}^{ \pm} \cdot \widehat{\mathbf{u}}_{ \pm}^{2} d s_{Z} \\
& \mathscr{E}_{B}^{\varepsilon}\left(\overline{\mathbf{u}}^{\varepsilon}\right)=\varepsilon^{-1} \mathscr{E}_{B}^{-1}\left(\overline{\mathbf{u}}^{0}\right)+\mathscr{C}_{B}^{0}\left(\overline{\mathbf{u}}^{0}, \overline{\mathbf{u}}^{1}\right)+\varepsilon_{\mathscr{E}}^{\mathscr{C}}\left(\overline{\mathbf{u}}^{0}, \overline{\mathbf{u}}^{1}, \overline{\mathbf{u}}^{2}\right)+\varepsilon^{2} \mathscr{E}_{B}^{2}\left(\overline{\mathbf{u}}^{0}, \overline{\mathbf{u}}^{1}, \overline{\mathbf{u}}^{2}, \overline{\mathbf{u}}^{3}\right)+o\left(\varepsilon^{2}\right), \\
& \mathscr{E}_{B}^{-1}\left(\overline{\mathbf{u}}^{0}\right)=\frac{1}{2} \int{ }_{\bar{B}} \mathbf{K}_{0}^{33}\left(\overline{\mathbf{u}}_{, 3}^{0}\right) \cdot \overline{\mathbf{u}}_{, 3}^{0} d z \\
& \mathscr{E}_{B}^{0}\left(\overline{\mathbf{u}}^{0}, \overline{\mathbf{u}}^{1}\right)=\frac{1}{2} \int \bar{B}_{\bar{B}}\left(2 \mathbf{K}_{0}^{33}\left(\overline{\mathbf{u}}_{3,}^{0}\right) \cdot \overline{\mathbf{u}}_{3}^{1}+\mathbf{K}_{1}^{33}\left(\overline{\mathbf{u}}_{, 3}^{0}\right) \cdot \overline{\mathbf{u}}_{, 3}^{0}+2 \mathbf{K}_{0}^{\alpha 3}\left(\overline{\mathbf{u}}_{, \alpha}^{0}\right) \cdot \overline{\mathbf{u}}_{3,}^{0}\right) d z, \\
& \mathscr{E}_{B}^{1}\left(\overline{\mathbf{u}}^{0}, \overline{\mathbf{u}}^{1}, \overline{\mathbf{u}}^{2}\right)=\frac{1}{2} \int \bar{B}_{\bar{B}}\left(\mathbf{K}_{0}^{33}\left(\overline{\mathbf{u}}_{, 3}^{1}\right) \cdot \overline{\mathbf{u}}_{, 3}^{1}+2 \mathbf{K}_{0}^{33}\left(\overline{\mathbf{u}}_{, 3}^{0}\right) \cdot \overline{\mathbf{u}}_{, 3}^{2}+2 \mathbf{K}_{1}^{33}\left(\overline{\mathbf{u}}_{, 3}^{0}\right) \cdot \overline{\mathbf{u}}_{, 3}^{1}+2 \mathbf{K}_{0}^{\alpha 3}\left(\overline{\mathbf{u}}_{, \alpha}^{0}\right) \cdot \overline{\mathbf{u}}_{, 3}^{1}+2 \mathbf{K}_{0}^{\alpha 3}\left(\overline{\mathbf{u}}_{, \alpha}^{1}\right) \cdot \overline{\mathbf{u}}_{, 3}^{0}+2 \mathbf{K}_{1}^{\alpha 3}\left(\overline{\mathbf{u}}_{, \alpha}^{0}\right) \cdot \overline{\mathbf{u}}_{, 3}^{0}\right. \\
& \left.+\mathbf{K}_{0}^{\alpha \beta}\left(\overline{\mathbf{u}}_{, \alpha}^{0}\right) \cdot \overline{\mathbf{u}}_{, \beta}^{0}\right) d z
\end{aligned}
$$




$$
\begin{aligned}
& \mathscr{E}_{B}^{2}\left(\overline{\mathbf{u}}^{0}, \overline{\mathbf{u}}^{1}, \overline{\mathbf{u}}^{2}, \overline{\mathbf{u}}^{3}\right)=\frac{1}{2} \int \frac{\bar{B}}{2}\left(2 \mathbf{K}_{0}^{33}\left(\overline{\mathbf{u}}_{, 3}^{0}\right) \cdot \overline{\mathbf{u}}_{, 3}^{3}+2 \mathbf{K}_{0}^{33}\left(\overline{\mathbf{u}}_{, 3}^{1}\right) \cdot \overline{\mathbf{u}}_{, 3}^{2}\right. \\
+ & \mathbf{K}_{0}^{\alpha \beta}\left(\overline{\mathbf{u}}_{, \alpha}^{0}\right) \cdot \overline{\mathbf{u}}_{, \beta}^{0}+\mathbf{K}_{1}^{33}\left(\overline{\mathbf{u}}_{, 3}^{1}\right) \cdot \overline{\mathbf{u}}_{, 3}^{1}+2 \mathbf{K}_{1}^{33}\left(\overline{\mathbf{u}}_{, 3}^{0}\right) \cdot \overline{\mathbf{u}}_{, 3}^{2}+2 \mathbf{K}_{0}^{\alpha 3}\left(\overline{\mathbf{u}}_{, \alpha}^{1}\right) \cdot \overline{\mathbf{u}}_{, 3}^{1} \\
& +\mathbf{K}_{0}^{\alpha \beta}\left(\overline{\mathbf{u}}_{, \alpha}^{1}\right) \cdot \overline{\mathbf{u}}_{, \beta}^{0}+2 \mathbf{K}_{1}^{\alpha 3}\left(\overline{\mathbf{u}}_{, \alpha}^{0}\right) \cdot \overline{\mathbf{u}}_{, 3}^{1}+2 \mathbf{K}_{1}^{\alpha 3}\left(\overline{\mathbf{u}}_{, \alpha}^{1}\right) \cdot \overline{\mathbf{u}}_{, 3}^{0} \\
& \left.+\mathbf{K}_{0}^{\alpha \beta}\left(\overline{\mathbf{u}}_{, \alpha}^{0}\right) \cdot \overline{\mathbf{u}}_{, \beta}^{1}+2 \mathbf{K}_{0}^{\alpha 3}\left(\overline{\mathbf{u}}_{, \alpha}^{0}\right) \cdot \overline{\mathbf{u}}_{, 3}^{2}+2 \mathbf{K}_{0}^{\alpha 3}\left(\overline{\mathbf{u}}_{, \alpha}^{2}\right) \cdot \overline{\mathbf{u}}_{, 3}^{0}\right) d z
\end{aligned}
$$

In conclusion, the Lagrangian is expanded as follows

$$
\begin{aligned}
\breve{L}^{\varepsilon}\left(\widehat{\mathbf{u}}^{\varepsilon}\right)= & \varepsilon^{-1} \mathscr{L}^{-1}\left(\overline{\mathbf{u}}^{0}\right)+\mathscr{L}^{0}\left(\dot{\widehat{\mathbf{u}}}_{ \pm}^{0}, \widehat{\mathbf{u}}_{ \pm}^{0}, \overline{\mathbf{u}}^{0}, \overline{\mathbf{u}}^{1}\right) \\
& \left.+\varepsilon \mathscr{L}^{1} \dot{\hat{\mathbf{u}}}_{ \pm}^{0}, \dot{\hat{\mathbf{u}}}_{ \pm}^{1}, \dot{\mathbf{u}}_{ \pm}^{0}, \widehat{\mathbf{u}}_{ \pm}^{0}, \widehat{\mathbf{u}}_{ \pm}^{1}, \overline{\mathbf{u}}^{0}, \overline{\mathbf{u}}^{1}, \overline{\mathbf{u}}^{2}\right) \\
& \left.+\varepsilon^{2} \mathscr{L}^{2} \dot{\hat{\mathbf{u}}}_{ \pm}^{1}, \dot{\mathbf{u}}_{ \pm}^{0}, \dot{\mathbf{u}}_{ \pm}^{1}, \widehat{\mathbf{u}}_{ \pm}^{0}, \widehat{\mathbf{u}}_{ \pm}^{1}, \widehat{\mathbf{u}}_{ \pm}^{2}, \overline{\mathbf{u}}^{0}, \overline{\mathbf{u}}^{1}, \overline{\mathbf{u}}^{2}, \overline{\mathbf{u}}^{3}\right) \\
& +o\left(\varepsilon^{2}\right) .
\end{aligned}
$$

In order to minimize the Lagrangian, we suppose that it is possible to minimize each term in the expansion (32) independently, i.e. the Lagrange-Hamilton's principle on $\breve{\mathscr{L}}^{\varepsilon}$ is assumed to be equivalent to

$\delta \int_{t_{1}}^{t_{2}} \mathscr{L}^{i} d t=0, i=-1,0,1,2$.

A justification of this assumption relies on previous results obtained in the static case, where it has been shown that it is sufficient to consider only the minimization of the highest order energy term to derive all the Euler-Lagrange equations governing the problem (Rizzoni et al., 2014).

We recall that this last equation can be written as

$\frac{d}{d t} \frac{\partial}{\partial \dot{\mathbf{q}}_{k}} \mathscr{L}^{i}-\frac{\partial}{\partial \mathbf{q}_{k}} \mathscr{L}^{i}=0, i=-1,0,1,2$,

where $\mathbf{q}_{k} \in\left\{\overline{\mathbf{u}}^{0}, \overline{\mathbf{u}}^{1}, \overline{\mathbf{u}}^{2}, \overline{\mathbf{u}}^{3}, \widehat{\mathbf{u}}_{+}^{0}, \widehat{\mathbf{u}}_{+}^{1}, \widehat{\mathbf{u}}_{+}^{2}\right\}$ are the generalized coordinates. The generalized coordinates defined on the rescaled adhesive, $\overline{\mathbf{u}}^{0}, \overline{\mathbf{u}}^{1}, \overline{\mathbf{u}}^{2}, \overline{\mathbf{u}}^{3}$, are assumed to belong to $\bar{V}^{i}$, subsets of $H^{1}\left(\left[t_{1}, t_{2}\right] ; H^{1}(\bar{B})\right)$, the set of vector-valued functions continuous and differentiable as many times as necessary on $\left[t_{1}, t_{2}\right] \times \bar{B}$ satisfying the boundary and initial conditions:

$$
\begin{aligned}
& \bar{V}^{i}=\left\{\overline{\mathbf{u}} \in H^{1}\left(\left[t_{1}, t_{2}\right] ; H^{1}\left(\bar{B} ; R^{3}\right)\right) ; \overline{\mathbf{u}}=\widehat{\mathbf{u}}^{i} \text { on } S^{ \pm} \times\left[t_{1}, t_{2}\right],\right. \\
& \left.\overline{\mathbf{u}}=\overline{\mathbf{U}}^{i} \text { in }\left\{t_{1}\right\} \times \bar{B}, \dot{\mathbf{u}}=\overline{\mathbf{v}}^{i} \text { in }\left\{t_{1}\right\} \times \bar{B}\right\}, \quad i=1,2, \ldots
\end{aligned}
$$

with $S^{ \pm}=S \times\left\{\frac{ \pm}{2} 1 / 2\right\}$. In the rescaled adherents, the displacement fields $\widehat{\mathbf{u}}_{ \pm}^{0}, \widehat{\mathbf{u}}_{ \pm}^{1}, \widehat{\mathbf{u}}_{ \pm}^{2}$ are assumed to belong to the following sets of kinematically admissible displacements

$$
\begin{aligned}
\widehat{V}^{i} & =\left\{\widehat{\mathbf{u}} \in H^{1}\left(\left[t_{1}, t_{2}\right] ; H^{1}\left(\widehat{\Omega}_{+} \cup \widehat{\Omega}_{-} ; R^{3}\right)\right): \widehat{\mathbf{u}}\right. \\
& =0 \text { on }\left[t_{1}, t_{2}\right] \times \widehat{S}_{u}^{ \pm},
\end{aligned}
$$

$$
\begin{aligned}
& \widehat{\mathbf{u}}=\overline{\mathbf{u}}^{i} \text { on }\left[t_{1}, t_{2}\right] \times S^{ \pm}, \quad \widehat{\mathbf{u}}=\widehat{\mathbf{U}}^{i} \text { in }\left\{t_{1}\right\} \times\left(\widehat{\Omega}_{+} \cup \widehat{\Omega}_{-}\right), \\
& \left.\dot{\widehat{\mathbf{u}}}=\widehat{\mathbf{v}}^{i} \text { in }\left\{t_{1}\right\} \times\left(\widehat{\Omega}_{+} \cup \widehat{\Omega}_{-}\right)\right\}, \quad i=1,2, \ldots
\end{aligned}
$$

Note that in the definition of sets $\bar{V}^{i}$ and $\widehat{V}^{i}$ the initial conditions have be expanded using notations proposed in (27).

Minimization of $\mathscr{L}^{-1}$ At order -1 , the Lagrangian is

$$
\mathscr{L}^{-1}\left(\overline{\mathbf{u}}^{0}\right)=-\frac{1}{2} \int_{\bar{B}} \mathbf{K}_{0}^{33}\left(\overline{\mathbf{u}}_{, 3}^{0}\right) \cdot \overline{\mathbf{u}}_{, 3}^{0} d z .
$$

In view of equation (34), one obtains

$\int_{\bar{B}} \mathbf{K}_{0}^{33}\left(\overline{\mathbf{u}}_{, 3}^{0}\right) \cdot \mathbf{v} d z=0, \forall \mathbf{v} \in \bar{V}^{0}$

which, if $\mathbf{K}_{0}^{33} \neq 0$, implies (Lebon and Rizzoni, 2010, 2011)

$\overline{\mathbf{u}}_{, 3}^{0}=0$ in $\left[t_{1}, t_{2}\right] \times \bar{B}$.

We take [ ${ }^{*}$ to denote the jump along $\bar{B}$ in the third direction, i.e. $[\mathbf{f}]^{*}=\mathbf{f}\left(t, z_{1}, z_{2}, \frac{1}{2}\right)-\mathbf{f}\left(t, z_{1}, z_{2},-\frac{1}{2}\right)$. Thus, we have

$\left[\overline{\mathbf{u}}^{0}\right]^{*}=0$ in $\left[t_{1}, t_{2}\right] \times S$.

Note, that the condition of perfect adherence between the adhesive and the adherents, at order 0 , gives

$\mathbf{u}^{0}\left(t, x_{1}, x_{2}, 0^{ \pm}\right)=\widehat{\mathbf{u}}_{ \pm}^{0}\left(t, z_{1}, z_{2}, \pm \frac{1}{2}\right)=\overline{\mathbf{u}}^{0}\left(t, z_{1}, z_{2}, \pm \frac{1}{2}\right)$

$$
\sigma_{i 3}^{0}\left(t, x_{1}, x_{2}, 0^{ \pm}\right)=\widehat{\sigma}_{i 3}^{0}\left(t, z_{1}, z_{2}, \pm \frac{1}{2}\right)=\bar{\sigma}_{i 3}^{0}\left(t, z_{1}, z_{2}, \pm \frac{1}{2}\right), \quad i
$$$$
=1,2,3 \text {, }
$$

where the Taylor series along the $x_{3}$ - direction of $\mathbf{u}^{\varepsilon}$ and $\boldsymbol{\sigma}^{\varepsilon}$ have been taken into account together with the asymptotic expansions (9). Thus,

$$
\left[\mathbf{u}^{0}\right]=0 \text { in }\left[t_{1}, t_{2}\right] \times S \text {. }
$$

Here, the symbol [] is taken to denote the jump across $S$ in the limit configuration.

Minimization of $\mathscr{D}^{0}$ In view of (40) the Lagrangian at order 0 simplifies as

$$
\begin{gathered}
\mathscr{L}^{0}=\frac{1}{2} \int_{\widehat{\Omega}_{ \pm}} \rho_{ \pm}\left\|\widehat{\mathbf{u}}_{ \pm}^{0}\right\|^{2} d z-\frac{1}{2} \int_{\widehat{\Omega}_{ \pm}} \mathbf{a}_{ \pm}\left(\mathbf{e}\left(\widehat{\mathbf{u}}_{ \pm}^{0}\right)\right) \cdot \mathbf{e}\left(\widehat{\mathbf{u}}_{ \pm}^{0}\right) d z \\
+\int_{\widehat{\Omega}_{ \pm}} \widehat{\mathbf{f}}^{ \pm} \cdot \widehat{\mathbf{u}}_{ \pm}^{0} d z+\int_{\widehat{S}_{g}} \widehat{\mathbf{g}}^{ \pm} \cdot \widehat{\mathbf{u}}_{ \pm}^{0} d s_{z} .
\end{gathered}
$$

Thus, equations (34) and (40) give after integration by parts 


$$
\begin{aligned}
& -\int_{\widehat{\Omega}_{ \pm}}\left(\rho_{ \pm} \ddot{\hat{u}}_{ \pm}^{0}-\operatorname{div}\left(\mathbf{a}_{ \pm}\left(\mathbf{e}\left(\widehat{\mathbf{u}}_{ \pm}^{0}\right)\right)\right)-\widehat{\mathbf{f}}^{ \pm}\right) \cdot \mathbf{v} d z-\int_{\widehat{S}_{g}}\left(\mathbf{a}_{ \pm}\left(\mathbf{e}\left(\widehat{\mathbf{u}}_{ \pm}^{0}\right)\right) \mathbf{n}\right. \\
& \left.-\widehat{\mathbf{g}}^{ \pm}\right) \cdot \mathbf{v} d s_{z} \\
& +\int_{S^{+}} \mathbf{a}_{+}\left(\mathbf{e}\left(\widehat{\mathbf{u}}_{+}^{0}\right)\right) \mathbf{e}_{3} \cdot \mathbf{v} d s_{z}-\int_{S^{-}} \mathbf{a}_{-}\left(\mathbf{e}\left(\widehat{\mathbf{u}}_{-}^{0}\right)\right) \mathbf{e}_{3} \cdot \mathbf{v} d s_{z} \\
& \quad=0 \quad \forall \mathbf{v} \in \widehat{V}^{0} .
\end{aligned}
$$

Using standard arguments and taking into account the constitutive equations in the adherents and the initial condition, we obtain the classical equations of motion posed only in the adherents

$$
\left\{\begin{array}{cc}
\widehat{\sigma}_{i j, j}^{0}+\widehat{f}_{i}^{ \pm}=\rho_{ \pm} \ddot{\widehat{u}}_{i}^{0} & \text { in }\left[t_{1}, t_{2}\right] \times \widehat{\Omega}_{ \pm}, \\
\widehat{\sigma}_{i j}^{0} n_{j}=\widehat{g}_{i}^{ \pm} & \text {on }\left[t_{1}, t_{2}\right] \times \widehat{S}_{g}^{ \pm}, \\
\widehat{u}_{i}^{0}=0 & \text { on }\left[t_{1}, t_{2}\right] \times \widehat{S}_{u}^{ \pm}, \\
\widehat{\sigma}_{i j}^{0}=a_{i j h k}^{ \pm} e_{h k}\left(u^{0}\right) & \text { in }\left[t_{1}, t_{2}\right] \times \widehat{\Omega}_{ \pm}, \\
\widehat{u}_{i}^{0}=\widehat{U}_{i}^{0} & \text { on }\left\{t_{1}\right\} \times \widehat{\Omega}_{ \pm}, \\
\dot{\hat{u}}_{i}^{0}=\widehat{v}_{i}^{0} & \text { on }\left\{t_{1}\right\} \times \widehat{\Omega}_{ \pm} .
\end{array}\right.
$$

The term $\int_{S^{+}} \hat{\boldsymbol{\sigma}}^{0} \mathbf{e}_{3} \cdot \mathbf{v} d s_{z}-\int_{S^{-}} \hat{\boldsymbol{\sigma}}^{0} \mathbf{e}_{3} \cdot \mathbf{v} d s_{z}=0$ implies that $\int_{S}\left[\hat{\boldsymbol{\sigma}}^{0}\right]^{*} \mathbf{e}_{3} \cdot \mathbf{v} d s_{z}$ and the continuity condition (42) gives $\left[\hat{\boldsymbol{\sigma}}^{0}\right]^{*} \mathbf{e}_{3}=0$. Thus, the problem at order 0 in the limit configuration obtained for $\varepsilon \rightarrow 0$ can be written as

$$
\left\{\begin{array}{cc}
\sigma_{i j, j}^{0}+f_{i}^{ \pm}=\rho_{ \pm} \ddot{u}_{i}^{0} & \text { in }\left[t_{1}, t_{2}\right] \times \Omega_{ \pm}, \\
\sigma_{i j}^{0} n_{j}=\widehat{g}_{i}^{ \pm} & \text {on }\left[t_{1}, t_{2}\right] \times S_{g}^{ \pm}, \\
u_{i}^{0}=0 & \text { on }\left[t_{1}, t_{2}\right] \times S_{u}^{ \pm}, \\
\sigma_{i j}^{0}=a_{i j h k}^{ \pm} e_{h k}\left(u^{0}\right) & \text { in }\left[t_{1}, t_{2}\right] \times \Omega_{ \pm}, \\
{\left[u_{i}^{0}\right]=0} & \text { on }\left[t_{1}, t_{2}\right] \times S, \\
{\left[\sigma_{i 3}^{0}\right]=0} & \text { on }\left[t_{1}, t_{2}\right] \times S, \\
u_{i}^{0}=U_{i}^{0} & \text { on }\left\{t_{1}\right\} \times \widehat{\Omega}_{ \pm}, \\
\dot{u}_{i}^{0}=v_{i}^{0} & \text { on }\left\{t_{1}\right\} \times \widehat{\Omega}_{ \pm},
\end{array}\right.
$$

where $\Omega_{ \pm}=\left\{\left(x_{1}, x_{2}, x_{3}\right) \in \Omega: \pm x_{3}>0\right\}$. As a conclusion, a dynamic problem with perfect adhesion between the adherents is obtained at the order 0 .

Minimization of $\mathscr{L}^{1}$ Using the relations obtained in the previous sections, the Lagrangian at order 1 becomes

$$
\begin{aligned}
& \mathscr{L}^{1}=\int_{S^{+}} \widehat{\boldsymbol{\sigma}}^{0} \mathbf{e}_{3} \cdot \widehat{\mathbf{u}}^{1} d s_{z}-\int_{S^{-}} \widehat{\boldsymbol{\sigma}}^{0} \mathbf{e}_{3} \cdot \widehat{\mathbf{u}}^{1} d s_{z} \\
& -\int_{\bar{B}}\left(\frac{1}{2} \mathbf{K}_{0}^{33}\left(\overline{\mathbf{u}}_{, 3}^{1}\right) \cdot \overline{\mathbf{u}}_{, 3}^{1}+\mathbf{K}_{0}^{\alpha 3}\left(\overline{\mathbf{u}}_{, \alpha}^{0}\right) \cdot \overline{\mathbf{u}}_{, 3}^{1}\right) d z,
\end{aligned}
$$

up to terms in $\widehat{\mathbf{u}}^{0}, \overline{\mathbf{u}}^{0}$ which are considered constant because the fields $\widehat{\mathbf{u}}^{0}, \overline{\mathbf{u}}^{0}$ are determined at the lower order by solving (47). Thus,

$$
\begin{aligned}
& \int_{S} \hat{\boldsymbol{\sigma}}^{0} \mathbf{e}_{3} \cdot[\mathbf{v}]^{*} d s_{z}-\int_{\bar{B}}\left(\mathbf{K}_{0}^{33}\left(\overline{\mathbf{u}}_{, 3}^{1}\right)+\mathbf{K}_{0}^{\alpha 3}\left(\overline{\mathbf{u}}_{, \alpha}^{0}\right)\right. \\
& \quad \times) \cdot \mathbf{w}_{, 3} d z \quad \forall \mathbf{v} \in \widehat{V}^{1}, \mathbf{w} \in \bar{V}^{1} .
\end{aligned}
$$

This last equation and the condition of perfect adherence between the adhesive and the adherents at order 1 in the rescaled configuration (see the last equality in (52) below) imply that $\overline{\mathbf{u}}_{3,}^{1}$ is independent of $z_{3}$ or, equivalently, that the jump $\left[\overline{\mathbf{u}}^{1}\right]^{*}$ is independent of $z_{3}$ and it is given by

$$
\left[\overline{\mathbf{u}}^{1}\right]^{*}=\left(\mathbf{K}_{0}^{33}\right)^{-1}\left(\hat{\boldsymbol{\sigma}}^{0} \mathbf{e}_{3}-\mathbf{K}_{0}^{\alpha 3} \overline{\mathbf{u}}_{, \alpha}^{0}\right) .
$$

Note that the inertial forces have no influence on this last result.

The conditions of perfect adherence between the adhesive and the adherents, written at order 1 for the limit and the rescaled configurations, give

$$
\begin{aligned}
\mathbf{u}^{1}\left(t, x_{1}, x_{2}, 0^{ \pm}\right) \pm \frac{1}{2} \mathbf{u}_{, 3}^{0}\left(t, x_{1}, x_{2}, 0\right) & =\widehat{\mathbf{u}}_{ \pm}^{1}\left(t, z_{1}, z_{2}, \pm \frac{1}{2}\right) \\
& =\overline{\mathbf{u}}^{1}\left(t, z_{1}, z_{2}, \pm \frac{1}{2}\right) \\
\boldsymbol{\sigma}_{i 3}^{1}\left(t, x_{1}, x_{2}, 0^{ \pm}\right) \pm \frac{1}{2} \sigma_{i 3,3}^{0}\left(t, x_{1}, x_{2}, 0\right) & =\widehat{\boldsymbol{\sigma}}_{i 3}^{1}\left(t, z_{1}, z_{2}, \pm \frac{1}{2}\right) \\
= & \bar{\sigma}_{i 3}^{1}\left(t, z_{1}, z_{2}, \pm \frac{1}{2}\right)
\end{aligned}
$$

where $i=1,2,3$ and the Taylor series along the $x_{3}$ - direction of $\mathbf{u}^{\varepsilon}$ and $\sigma^{\varepsilon}$ have been taken into account together with the asymptotic expansions (9). Thus,

$$
\left[\mathbf{u}^{1}\right]=\left(\mathbf{K}_{0}^{33}\right)^{-1}\left(\boldsymbol{\sigma}^{0} \mathbf{e}_{3}-\mathbf{K}_{0}^{\alpha 3} \mathbf{u}_{, \alpha}^{0}\right)-<\mathbf{u}_{, 3}^{0}>
$$

where $<\mathbf{f}>=\frac{1}{2}\left(\mathbf{f}\left(0^{+}\right)+\mathbf{f}\left(0^{-}\right)\right.$.

Minimization of $\mathscr{L}^{2}$ Using the relations obtained in the previous sections, the Lagrangian at order 2 can be written as

$$
\begin{aligned}
& \left.\mathscr{L}^{2}=\int_{\widehat{\Omega}_{ \pm}} \rho_{ \pm} \frac{1}{2}\left\|\dot{\widehat{\mathbf{u}}}_{ \pm}^{1}\right\|^{2}\right) d z+\int_{\bar{B}}\left(\zeta^{0}\left(\dot{\overline{\mathbf{u}}}^{\mathbf{0}} \cdot \dot{\overline{\mathbf{u}}}^{1}\right)\right) d z \\
& -\frac{1}{2} \int_{\widehat{\Omega}_{ \pm}} \mathbf{a}_{ \pm}\left(\mathbf{e}\left(\widehat{\mathbf{u}}_{ \pm}^{1}\right)\right) \cdot \mathbf{e}\left(\widehat{\mathbf{u}}_{ \pm}^{1}\right) d z-\frac{1}{2} \int_{\bar{B}}\left(2 \mathbf{K}_{0}^{33}\left(\overline{\mathbf{u}}_{, 3}^{1}\right) \cdot \overline{\mathbf{u}}_{, 3}^{2}\right. \\
& +2 \mathbf{K}_{0}^{\alpha 3}\left(\overline{\mathbf{u}}_{, \alpha}^{1}\right) \cdot \overline{\mathbf{u}}_{, 3}^{1}+\mathbf{K}_{0}^{\alpha \beta}\left(\overline{\mathbf{u}}_{, \alpha}^{1}\right) \cdot \overline{\mathbf{u}}_{, \beta}^{0}+\mathbf{K}_{0}^{\alpha \beta}\left(\overline{\mathbf{u}}_{, \alpha}^{0}\right) \cdot \overline{\mathbf{u}}_{, \beta}^{1} \\
& \left.+2 \mathbf{K}_{0}^{\alpha 3}\left(\overline{\mathbf{u}}_{, \alpha}^{0}\right) \cdot \overline{\mathbf{u}}_{, 3}^{2}\right) d z,
\end{aligned}
$$

up to (constant) terms in $\widehat{\mathbf{u}}^{0}, \overline{\mathbf{u}}^{0}$. Note also that, in view of (51), the term $\overline{\mathbf{u}}_{3}^{1}$ is completely given in terms of $\overline{\mathbf{u}}^{0}$ and thus it can also be considered constant. Equations (34) and (3) applied to $\widehat{\mathbf{u}}_{ \pm}^{1}$ and $\overline{\mathbf{u}}^{1}$ give after integration by parts

$$
\begin{aligned}
& \int_{\widehat{\Omega}_{ \pm}}\left(\rho_{ \pm} \ddot{\widehat{\mathbf{u}}}_{ \pm}^{1}-\operatorname{div}\left(\mathbf{a}_{ \pm}\left(\mathbf{e}\left(\widehat{\mathbf{u}}_{ \pm}^{1}\right)\right)\right) \cdot \mathbf{v} d z-\int_{S^{+}} \widehat{\boldsymbol{\sigma}}^{1} \mathbf{e}_{3} \cdot \mathbf{v} d s_{z}\right. \\
& \quad+\int_{S^{-}} \widehat{\boldsymbol{\sigma}}^{1} \mathbf{e}_{3} \cdot \mathbf{v} d s_{z}+\int_{S_{g}^{ \pm}} \widehat{\boldsymbol{\sigma}}^{1} \mathbf{n} \cdot \mathbf{v} d s_{z}+\int_{\bar{B}} \zeta^{0} \bar{u}^{0} \cdot \mathbf{w} d z \\
& \quad+\int_{\bar{B}}\left(\mathbf{K}_{0}^{3 \alpha}\left(\overline{\mathbf{u}}_{, 3}^{1}\right)+\mathbf{K}_{0}^{\beta \alpha}\left(\overline{\mathbf{u}}_{, \beta}^{0}\right)\right) \cdot \mathbf{w}_{, \alpha} d z=0, \forall \mathbf{v} \in \widehat{V}^{1}, \forall \mathbf{w} \in \bar{V}^{1} .
\end{aligned}
$$

From latter equation, using standard arguments, one obtains 


$$
\left\{\begin{array}{cc}
\widehat{\sigma}_{i j . j}^{1}=\rho_{ \pm} \ddot{\widehat{u}}_{i}^{1} & \text { in }\left[t_{1}, t_{2}\right] \times \widehat{\Omega}_{ \pm}, \\
\widehat{\sigma}_{i j}^{1} n_{j}=0 & \text { on }\left[t_{1}, t_{2}\right] \times \widehat{S}_{g}^{ \pm}, \\
\widehat{u}_{i}^{1}=0 & \text { on }\left[t_{1}, t_{2}\right] \times \widehat{S}_{u}^{ \pm}, \\
\widehat{\sigma}_{i j}^{1}=a_{i j h k}^{ \pm} e_{h k}\left(\widehat{u}^{1}\right) & \text { in }\left[t_{1}, t_{2}\right] \times \widehat{\Omega}_{ \pm}, \\
\widehat{u}_{i}^{1}=\widehat{U}_{i}^{1} & \text { on }\left\{t_{1}\right\} \times \widehat{\Omega}_{ \pm}, \\
\hat{\widehat{u}}_{i}^{1}=\widehat{v}_{i}^{1} & \text { on }\left\{t_{1}\right\} \times \widehat{\Omega}_{ \pm} .
\end{array}\right.
$$

Using integration by parts (cf. the terms $\mathbf{w}_{, \alpha}$ ) and the continuity of the displacements at order 1 at the surfaces $S^{ \pm}$, one obtains

$\left[\widehat{\boldsymbol{\sigma}}^{1}\right]^{*} \mathbf{e}_{3}=\zeta^{0} \ddot{\mathbf{u}}^{0}-\mathbf{K}_{0}^{3 \alpha}\left(\overline{\mathbf{u}}_{, 3}^{1}\right)-\mathbf{K}_{0}^{\beta \alpha}\left(\overline{\mathbf{u}}_{, \beta}^{0}\right)$

which, in view of (51), becomes

$$
\begin{aligned}
{\left[\widehat{\boldsymbol{\sigma}}^{1}\right]^{*} \mathbf{e}_{3}=} & \zeta^{0} \ddot{\mathbf{u}}^{0}-\mathbf{K}_{0}^{3 \alpha}\left(\mathbf{K}_{0}^{33}\right)^{-1} \widehat{\boldsymbol{\sigma}}_{, \alpha}^{0} \mathbf{e}_{3}-\left(\mathbf{K}_{0}^{3 \alpha}\left(\mathbf{K}_{0}^{33}\right)^{-1} \mathbf{K}_{0}^{\beta 3}\right. \\
& \left.+\mathbf{K}_{0}^{\beta \alpha}\right) \overline{\mathbf{u}}_{, \alpha \beta}^{0} .
\end{aligned}
$$

Using the continuity conditions, the above conditions can be rephrased on the limit domain obtained as $\varepsilon \rightarrow 0$ : in the limit configuration can be rewritten as:

$$
\begin{aligned}
& {\left[\mathbf{u}^{\varepsilon}\right] \approx \varepsilon\left(\mathbf{K}^{33}\right)^{-1}\left(\left\langle\boldsymbol{\sigma}^{\varepsilon}\right\rangle \mathbf{e}_{3}-\mathbf{K}^{\alpha 3}<\mathbf{u}_{, \alpha}^{\varepsilon}>\right)-\varepsilon<\mathbf{u}_{, 3}^{\varepsilon}>,} \\
& {\left[\boldsymbol{\sigma}^{\varepsilon} \mathbf{e}_{3}\right] \approx \varepsilon \zeta^{\varepsilon}\left\langle\ddot{\mathbf{u}}^{\varepsilon}\right\rangle-\varepsilon \mathbf{K}^{3 \alpha}\left(\mathbf{K}^{33}\right)^{-1}<\boldsymbol{\sigma}_{, \alpha}^{\varepsilon}>\mathbf{e}_{3}} \\
& \quad-\varepsilon\left(\mathbf{K}^{3 \alpha}\left(\mathbf{K}^{33}\right)^{-1} \mathbf{K}^{\beta 3}+\mathbf{K}^{\beta \alpha}\right)<\mathbf{u}_{, \alpha \beta}^{\varepsilon}>-\varepsilon<\boldsymbol{\sigma}_{, 3}^{\varepsilon}>.
\end{aligned}
$$

Note the use of the average operator $<\cdot>$ introduced to take into account the discontinuity of the stress and displacement fields $\boldsymbol{\sigma}^{\varepsilon} \mathbf{i}_{3}$ and $\mathbf{u}^{\varepsilon}$ established at order one.

It is important to note that the imperfect interface model characterized by the relations (62) and (63) includes as special cases four widely used interface models: the perfect interface, the mass interface, the spring interface and the spring-mass interface.

To see this, we first consider the case in which the interface stiffness is much lower than that of the adherent stiffness. This case can be formulated as follows:

$\mathbf{K}^{j l}=\varepsilon \tilde{\mathbf{K}}^{j l}$,

where $\tilde{*}$ will denote quantities independent of $\varepsilon$. If $\zeta^{\varepsilon}=\tilde{\zeta}$, then

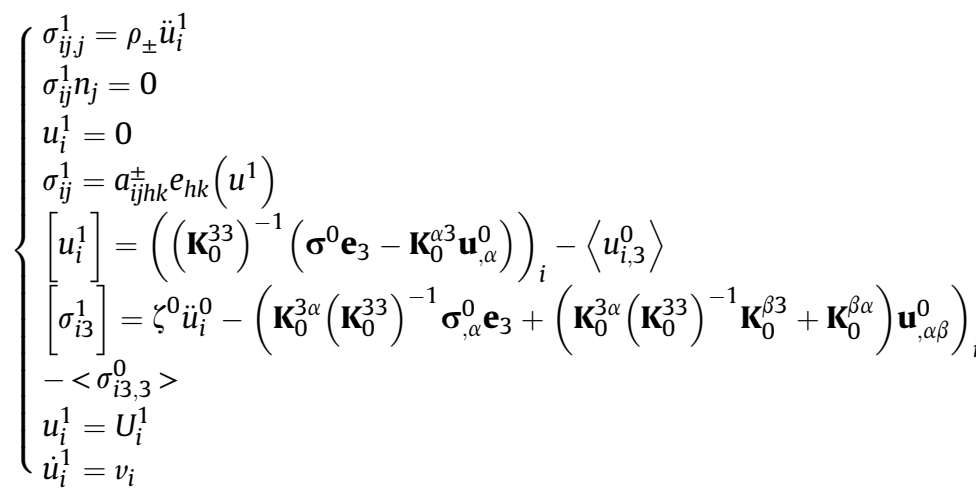

$$
\begin{aligned}
& \text { in }\left[t_{1}, t_{2}\right] \times \Omega_{ \pm}, \\
& \text {on }\left[t_{1}, t_{2}\right] \times S_{g}^{ \pm}, \\
& \text {on }\left[t_{1}, t_{2}\right] \times S_{\mathbf{u}}^{ \pm}, \\
& \text {in }\left[t_{1}, t_{2}\right] \times \Omega_{ \pm}, \\
& \text {on }\left[t_{1}, t_{2}\right] \times S, \\
& \\
& \text { on }\left[t_{1}, t_{2}\right] \times S, \\
& \text { on }\left\{t_{1}\right\} \times \widehat{\Omega}_{ \pm}, \\
& \text {on }\left\{t_{1}\right\} \times \widehat{\Omega}_{ \pm} .
\end{aligned}
$$

Note that forces concentrated on the edge of $S$ emerge from the latter calculation

$\mathbf{F}=\mathbf{K}_{0}^{\beta \alpha}\left(\mathbf{u}_{, \beta}^{0}\right) n_{\alpha}+\mathbf{K}_{0}^{3 \alpha}\left[\mathbf{u}^{1}\right] n_{\alpha}$

In conclusion, a contact law of imperfect interface is obtained in (60), prescribing the jumps of the displacement and stress fields at the order 1 in terms of the corresponding fields solution of the elastodynamics problems at order 0 . Note that the inertial terms at order 0 enter the imperfect interface law.

\section{Condensed interface law}

The interface laws calculated at the order zero and at the order one can be condensed in a single formulation accounting for both contributions. Indeed, combining the asymptotic expansions (9), the matching conditions (42), (52), the fifth and the sixth of the equation (48) and the fifth and the sixth of the equation (60), the jumps of displacement and stress vector fields across the interface accounting for (64) in (62) and (63) it can be obtained that, to within an error of order $O(\varepsilon)$,

$$
\begin{aligned}
& \left\langle\boldsymbol{\sigma}^{\varepsilon}\right\rangle \mathbf{e}_{3} \approx \tilde{\mathbf{K}}^{33}\left[\mathbf{u}^{\varepsilon}\right], \\
& {\left[\boldsymbol{\sigma}^{\varepsilon} \mathbf{e}_{3}\right] \approx 0 .}
\end{aligned}
$$

These two equations represent the classical spring interface model. If $\zeta^{\varepsilon}=1 / \varepsilon \tilde{\zeta}$, then, accounting for (64) in (62) and (63), it can be deduced that, to within an error of order $O(\varepsilon)$,

$$
\begin{aligned}
& \left\langle\boldsymbol{\sigma}^{\varepsilon}\right\rangle \mathbf{e}_{3} \approx \tilde{\mathbf{K}}^{33}\left[\mathbf{u}^{\varepsilon}\right], \\
& {\left[\boldsymbol{\sigma}^{\varepsilon} \mathbf{e}_{3}\right] \approx \tilde{\zeta}<\ddot{\mathbf{u}}^{\varepsilon}>,}
\end{aligned}
$$

which represent the spring-mass interface model. In the case of a stiff interface, accounting for

$$
\mathbf{K}^{j l}=\tilde{\mathbf{K}}^{j l}
$$


and $\zeta^{\varepsilon}=\tilde{\zeta}$ in (62) and (63), it can be proved that, to within an error of order $O(\varepsilon)$,

$\left[\mathbf{u}^{\varepsilon}\right] \approx 0$,

$\left[\boldsymbol{\sigma}^{\varepsilon} \mathbf{e}_{3}\right] \approx 0$,

relations representing the behavior of a perfect interface. Finally, accounting for (69) and $\zeta^{\varepsilon}=1 / \varepsilon \tilde{\zeta}$ in (62) and (63), it can be shown that, to within an error of order $O(\varepsilon)$,

$\left[\mathbf{u}^{\varepsilon}\right] \approx 0$,

$\left[\boldsymbol{\sigma}^{\varepsilon} \mathbf{e}_{3}\right] \approx \tilde{\zeta}<\ddot{\mathbf{u}}^{\varepsilon}>$

which is a formulation of the mass interface model.

As a final remark, it is important that the presence of first order derivates of the displacement and stress vector fields make relations (62) and (63) nonlocal in character.

The implicit and nonlocal formulation given by relations (62) and (63) thus represents an enrichment of the classical interface models, obtained by taking into account high order effects. The formulation will be adopted in the application to phononic crystals proposed in the next Section.

\section{Band gaps in 1-D phononic crystals with imperfect interfaces}

Consider an one-dimensional phononic crystal, a periodic array of homogeneous isotropic adherent layers $\Omega_{ \pm}$of two types as in Fig. 2. The layers are separated by identical material interfaces whose behavior is modeled the contact conditions (62) and (63). The $x_{3}$ axis is perpendicular to the layers and the $\left(x_{1}, x_{2}\right)$ coordinate plane is parallel to the layers. The period of the array is $l=l_{+}+l_{-}$, where $l_{ \pm}$are the thicknesses of the two layers. The mass densities and the elastic constants of the two layers, taken to be isotropic, are denoted by $\rho_{ \pm}$and $\lambda_{ \pm}, \mu_{ \pm}$, respectively.

We consider the propagation along the $x_{3}-$ axis of timeharmonic (shear, SH, and longitudinal, P) elastic waves through the periodic array. The displacement in the material is assumed to have only a 1 - component for the shear wave and a 3 - component for the longitudinal wave. The two components inside the layers are denoted by $u_{ \pm}$and satisfy the motion equations

$u_{ \pm}^{\prime \prime}+\kappa^{2} \rho_{ \pm} u_{ \pm}=0, \quad x_{3} \in \Omega_{ \pm}^{n}, \quad n=0, \pm 1, \pm 2, \ldots$,

where

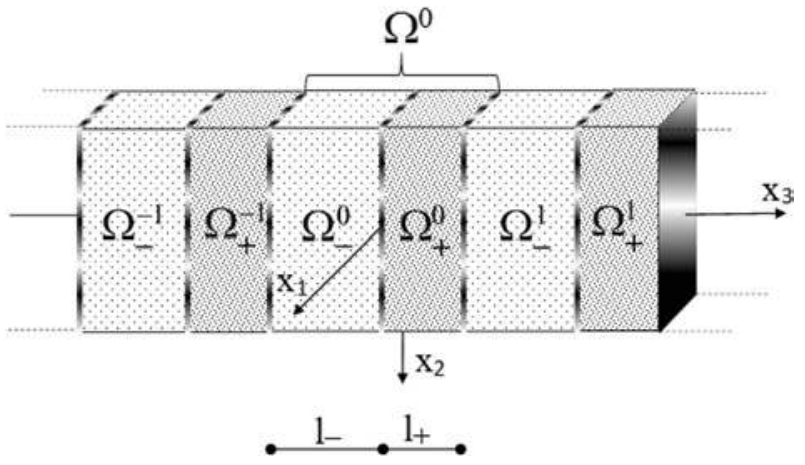

Fig. 2. Sketch of a 1D phononic crystal as a layered periodic structure obtained by alternating different layers separated by higher order imperfect interfaces. $\kappa_{ \pm}=\omega / c_{t}, \quad c_{t}=\sqrt{\mu_{ \pm} / \rho_{ \pm}}$

for SH waves and

$\kappa_{ \pm}=\omega / c_{l}, \quad c_{l}=\sqrt{\left(\lambda_{ \pm}+2 \mu_{ \pm}\right) / \rho_{ \pm}}$

for P waves. The symbol $\omega$ is the circular frequency and the prime in (74) denotes differentiation with respect to the $x_{3}$ - coordinate. The solutions of equation (74) are

$u_{ \pm}\left(x_{3}\right)=A_{ \pm} \exp ^{i \kappa_{ \pm} x_{3}}+B_{ \pm} \exp ^{-i \kappa_{ \pm} x_{3}}$,

with $A_{ \pm}, B_{ \pm}$constant coefficients. The stress inside the layers (the component $\sigma_{13}$ for SH waves and the component $\sigma_{33}$ for P waves) are

$\sigma\left(x_{3}, t\right)=\rho_{ \pm} c_{ \pm}^{2} u_{ \pm}^{\prime}=i \rho_{ \pm} c_{ \pm}^{2} \kappa_{ \pm}\left(A_{ \pm} \exp ^{i \kappa_{ \pm} x_{3}}-B_{ \pm} \exp ^{-i \kappa_{ \pm} x_{3}}\right)$.

The interfaces separating the different layers are described by the laws (62), (63), in which the matrix $\mathbf{K}$ is chosen to correspond to an isotropic behavior. Thus, denoted with $\lambda$ and $\mu$ the elastic constants entering the matrix $\mathbf{K}$, denoted with $\zeta$ the mass density of the interface and with $\varepsilon$ its (small) thickness, the contact conditions (62), (63) specialized to the case of propagation of SH and P waves reduce to

$[u]=\varepsilon\left(\rho_{-} c_{-}^{2} / \gamma-1\right) u_{-}^{\prime} / 2+\varepsilon\left(\rho_{+} c_{+}^{2} / \gamma-1\right) u_{+}^{\prime} / 2$,

$[\sigma]=-\varepsilon\left(\zeta \omega^{2}\left(u_{-}+u_{+}\right)+\rho_{-} c_{-}^{2} u^{\prime \prime}{ }_{-}+\rho_{+} c_{+}^{2} u^{\prime \prime}+\right) / 2$

where $\gamma=\mu$ for the SH wave (for which $u=u_{1}, \sigma=\sigma_{13}$ ) and $\gamma=\lambda+2 \mu$ for the P wave (for which $u=u_{3}, \sigma=\sigma_{33}$ ). In view of (77, $78)$, one has that $u_{{ }_{ \pm}}^{\prime}=\sigma_{ \pm} /\left(\rho_{ \pm} c_{ \pm}^{2}\right)$ and $u_{ \pm}^{\prime \prime}=-\kappa_{ \pm}^{2} u^{\prime}{ }_{ \pm}=-\rho_{ \pm} \omega^{2} u_{ \pm}^{\prime}$, thus the contact conditions $(79,80)$ can be rewritten as

$[u]=a_{-} \sigma_{-}+a_{+} \sigma_{+}$,

$[\sigma]=b_{-} u_{-}+b_{+} u_{+}$

with

$a_{ \pm}=\left(1 / \gamma-1 /\left(\rho_{ \pm} c_{ \pm}^{2}\right)\right) \varepsilon / 2$

$b_{ \pm}=-\left(\zeta-\rho_{ \pm}\right) \omega^{2} \varepsilon / 2$.

Solving the system of equations (81) and (82) with respect to $u_{+}$ and $\sigma_{+}$gives

$\left(\begin{array}{c}u_{+} \\ \sigma_{+}\end{array}\right)=\mathbf{T}^{\varepsilon}\left(\begin{array}{l}u_{-} \\ \sigma_{-}\end{array}\right)$

with

$\mathbf{T}^{\varepsilon}:=\left(\begin{array}{cc}1 & -a_{+} \\ -b_{+} & 1\end{array}\right)^{-1}\left(\begin{array}{cc}1 & a_{-} \\ b_{-} & 1\end{array}\right)$

The transfer matrix of the imperfect interface obeying the transmission conditions $(79,80)$.

Within layer $\Omega_{ \pm}^{n}$, it follows from (77) and (78) that the displacement $u_{ \pm}^{(n)}$ and the stress $\sigma_{ \pm}^{(n)}$ on the left and the right surface of the layer are related by 


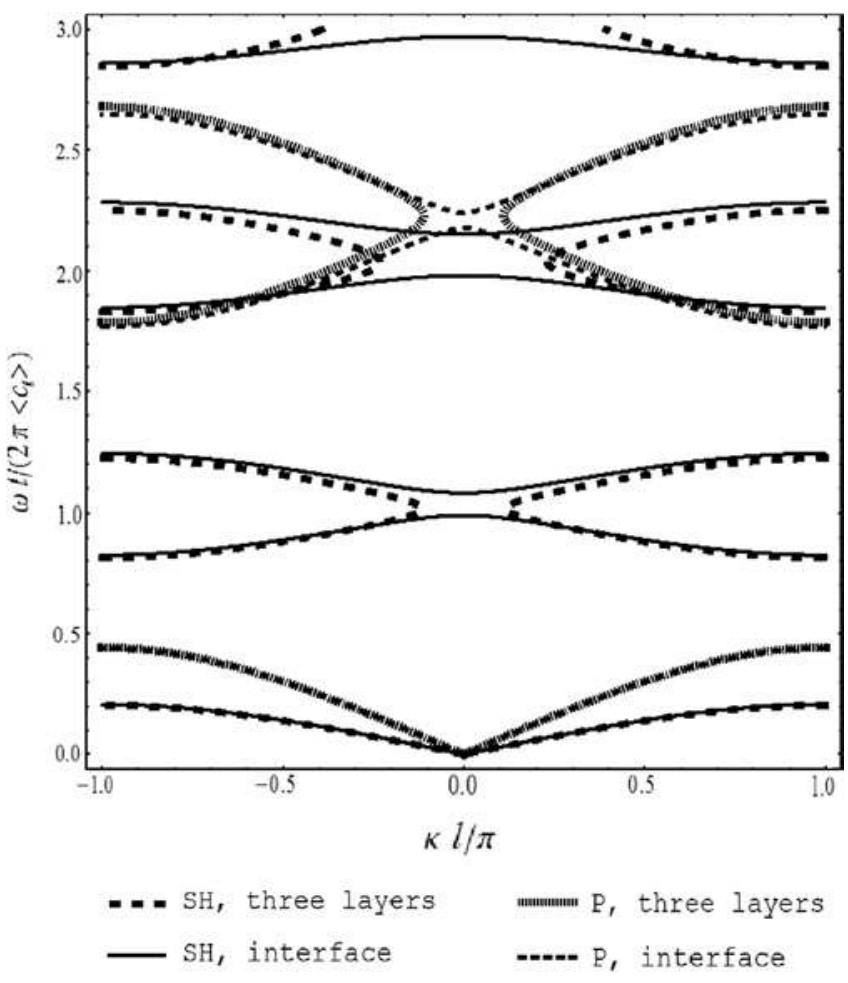

Fig. 3. Comparison between the band gaps for a one-dimensional phononic crystal made of three layers and the band gaps calculated for two layers separated by an interface. In the two situations, $\varepsilon$ is $1.0 \times 10^{-3} l$. The large dashed lines and the tiny dotted lines represent the dispersion curves of SH and $\mathrm{P}$ waves, respectively, for the crystal made of three layers. The solid lines and the small dashed lines represent the dispersion curves of $\mathrm{SH}$ and $\mathrm{P}$ waves, respectively, for the crystal made of two layers separated by the interface.

$\left(\begin{array}{c}u_{ \pm, R}^{(n)} \\ \sigma_{ \pm, R}^{(n)}\end{array}\right)=T_{ \pm}\left(\begin{array}{c}u_{ \pm, L}^{(n)} \\ \sigma_{ \pm, L}^{(n)}\end{array}\right)$

where the subscript $L$ and $R$ denote the left and right surface of each layer and

$T_{ \pm}:=\left(\begin{array}{cc}\cos \left(\kappa_{ \pm} l_{ \pm}\right) & \sin \left(\kappa_{ \pm} l_{ \pm}\right) /\left(\omega \rho_{ \pm} c_{ \pm}\right) \\ -\omega \rho_{ \pm} c_{ \pm} \sin \left(\kappa_{ \pm} l_{ \pm}\right) & \cos \left(\kappa_{ \pm} l_{ \pm}\right)\end{array}\right)$.

According to the Bloch-Floquet theory in a periodic structure, the displacement and the stress on the right surface of layer $\Omega_{+}^{n}$ are related to the displacement and the stress on the right surface of layer $\Omega_{+}^{n-1}$

$\left(\begin{array}{c}u_{ \pm, R}^{(n)} \\ \sigma_{ \pm, R}^{(n)}\end{array}\right)=\exp ^{i h l}\left(\begin{array}{c}u_{ \pm, L}^{(n-1)} \\ \sigma_{ \pm, L}^{(n-1)}\end{array}\right)$

with $h$ the Bloch parameter. Inserting equations (86)-(88) into equation (89) leads to the dispersion equation

$\operatorname{det}\left(T_{+} T^{\varepsilon} T_{-} T^{\varepsilon}-\exp ^{i h l} I\right)=0$.

We now numerically solve the dispersion equation and obtain the band gaps in the first Brillouin zone for a composite made of alternating layers of $\mathrm{Pb}$ and epoxy. We adopt the same values of the geometrical and material parameters as in (Zheng and Wei, 2009):

$$
\begin{aligned}
\lambda_{-} & =42.3 \mathrm{GPa}, \quad \mu_{-}=14.9 \mathrm{GPa} \quad \rho_{-}=11600 \mathrm{~kg} \mathrm{~m}^{-3}, \quad l_{-} \\
& =10 \mathrm{~mm},
\end{aligned}
$$

$$
\begin{aligned}
\lambda_{+} & =4.43 \mathrm{GPa}, \quad \mu_{+}=1.59 \mathrm{GPa} \quad \rho_{+}=1180 \mathrm{~kg} \mathrm{~m}^{-3}, \quad l_{+} \\
& =10 \mathrm{~mm} .
\end{aligned}
$$

The material properties of the thin layer are assumed to be as follows:

$$
\begin{aligned}
<\lambda> & :=1 / 2\left(\lambda_{-}+\lambda_{+}\right), \quad\left\langle\mu>:=1 / 2\left(\mu_{-}+\mu_{+}\right), \quad<\rho>:\right. \\
& =1 / 2\left(\rho_{-}+\rho_{+}\right) .
\end{aligned}
$$

To check the soundness of the interface law obtained in the present paper, Fig. 3 proposes a comparison between the band gaps of the phononic crystal obtained without using the interface law, i.e. by considering three different layers of finite thickness, and the bands gaps calculated for two layers separated by an interface obeying (62)-(63). For the comparison, $\varepsilon$ has been chosen equal to $1.0 \times 10^{-3} l$. Fig. 3 shows that the interface law yields accurate results for the chosen geometrical and material parameters.

We now discuss the effect of the parameters $\zeta, \lambda$ and $\mu$ of the imperfect interface model on the band gaps. To do so, three cases are considered with corresponding values of the geometrical and material parameters listed in Table 1 .

Case I considers the effect of an imperfect interface of increasing thickness, subcase $\varepsilon=0$ corresponding to perfect contact of the layers. Case II simulates an interface of increasing stiffness, starting from a soft interface behavior. Case III takes into account the effect of a density increase. Wave bands and band gaps of the phononic crystal with imperfect interface are shown in Fig. 4 for the case I, in Fig. 5 for the case II and in Fig. 6 for the case III. In the Figures, the solid lines and the dashed lines represent the dispersion curves of $\mathrm{SH}$ and $\mathrm{P}$ waves, respectively.

Due to the vanishing of the thickness $\varepsilon$, plot (a) of Fig. 4 represents the band structure of a phononic crystal with perfect contact between the layers. In this case, three band gaps for $\mathrm{SH}$ waves propagation and two band gaps for $\mathrm{P}$ waves propagation are observed in the considered frequency range. As the thickness of the interfacial layer increases, two new band gaps appear between the original first and second band gaps of SH waves. The new band gaps

Table 1

Chosen values of elastic coefficients, density and thickness of the imperfect interface model.

\begin{tabular}{llll}
\hline Case & $\varepsilon / l$ & $\lambda /\langle\lambda\rangle \mu /\langle\mu\rangle$ & $\zeta /\langle\rho\rangle$ \\
\hline I & 0 & 1.0 & 1.0 \\
& $1.0 \times 10^{-3}$ & & \\
& $5.0 \times 10^{-3}$ & & \\
& $1.0 \times 10^{-2}$ & & 1.0 \\
II & $1.0 \times 10^{-3}$ & $1.0 \times 10^{-2}$ & \\
& & $5.0 \times 10^{-2}$ & $1.0 \times 10^{-2}$ \\
& & $1.0 \times 10^{-1}$ & $1.0 \times 10^{-1}$ \\
& & $5.0 \times 10^{-1}$ & $1.0 \times 10^{+1}$ \\
\hline III & $1.0 \times 10^{-3}$ & & \\
& & & \\
& & &
\end{tabular}



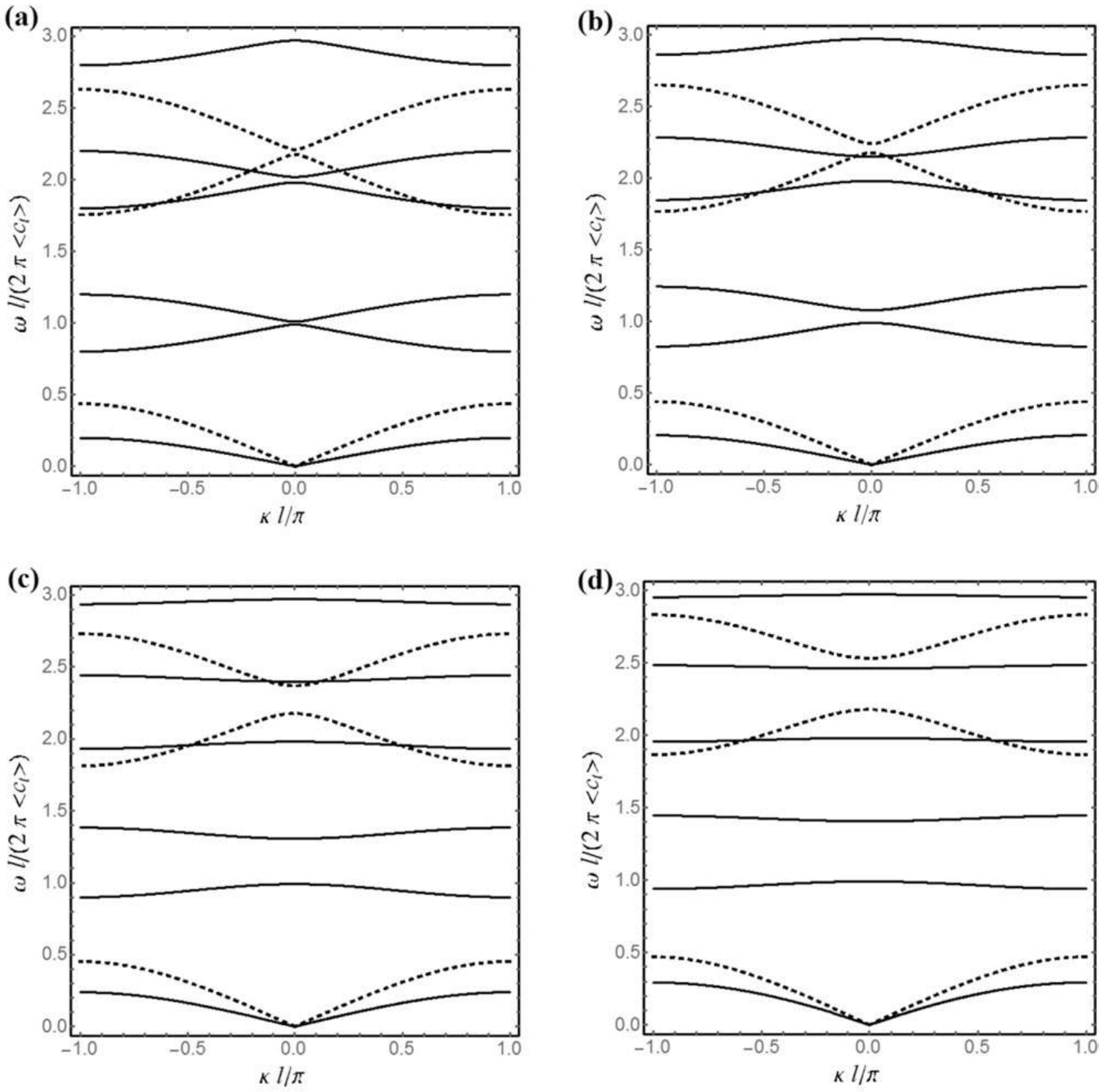

- SH, interface

\section{P, interface}

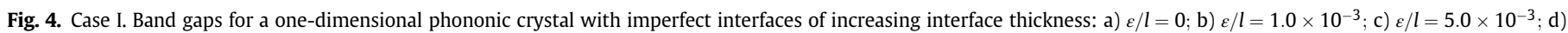
$\varepsilon / l=1.0 \times 10^{-2}$. The solid lines and the dashed lines represent the dispersion curves of SH and $\mathrm{P}$ waves, respectively.

become more and more wide as the thickness increases and the band structure gradually evolves from a propagating mode to a localized one. The occurrence of a new band gap is also observed for $P$ waves. As the interface stiffness decreases, the system is more dispersive, in agreement with the numerical results obtained in (Brito-Santana et al., 2015). A similar behavior is noted in Fig. 5 when the interface stiffness decreases moving from a hard interface behavior (d) to a softer one (a). The evolution of the band structure due to an interface of increasing density is shown in Fig. 6 and, again, new band gaps appear and the flat lines indicate localized modes.
The results presented in Figs. 4-6 are in agreement with the findings of (Zheng and Wei, 2009), where the classical interface models, the perfect interface, the mass interface, the spring interface and the spring-mass interface, are studied separately and then compared.

\section{Discussion and conclusion}

In this paper, a model of imperfect interface has been derived from the asymptotic study of a three phase composite with perfectly bonding conditions between two adherents and an 

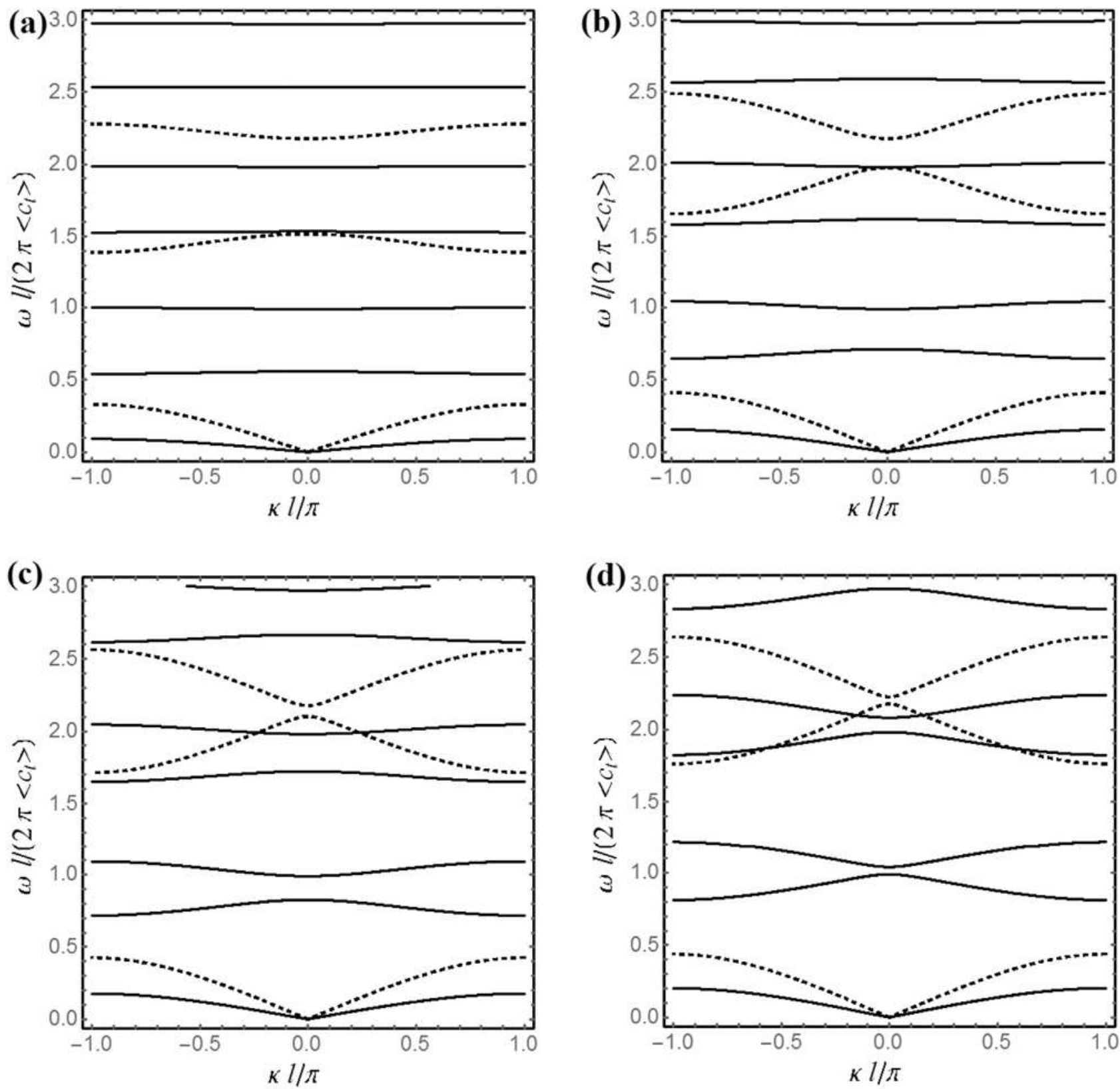

$\mathrm{SH}$, interface

\section{$P$, interface}

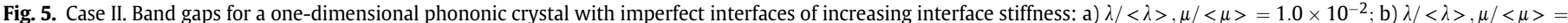

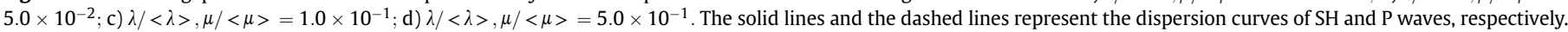

adhesive. It is shown that, if the stiffness of the glue is not too small, at the first order in the expansion (the order 0) a model of perfect interface is obtained. The expansion at the next levels yields a model of imperfect interface (at the order 1 ) taking into account the inertial terms from the lower order and the partial derivatives of the displacement and the stress vector fields. The laws obtained in the present paper generalize to the dynamic case the imperfect contact laws given in (Licht et al., 2013; Rizzoni et al., 2014) and represent an unification and extension of the four classical interface models, the perfect interface, the mass interface, the spring interface and the spring-mass interface.
In (Bövik, 1994), Bövik addresses the modeling of thin layers in both elastic and acoustic scattering problems. Here, we are interested on the first type of problems. In his derivation, the thin layer is replaced by single surface of discontinuity modeled by a set of boundary conditions of the order $\varepsilon$ Bövik, 1994, eqns. (35) and (36). Bövik's derivation is essentially based on a continuation of the field variables from the adherents into the thin layer. In other words, the thin layer is eliminated and it is replaced by an interface positioned at the location of $S$ with the two adherents being extended up to it As a consequence, the material properties of the adherents appear in the interface laws together with those of the thin layers and the 
(a)
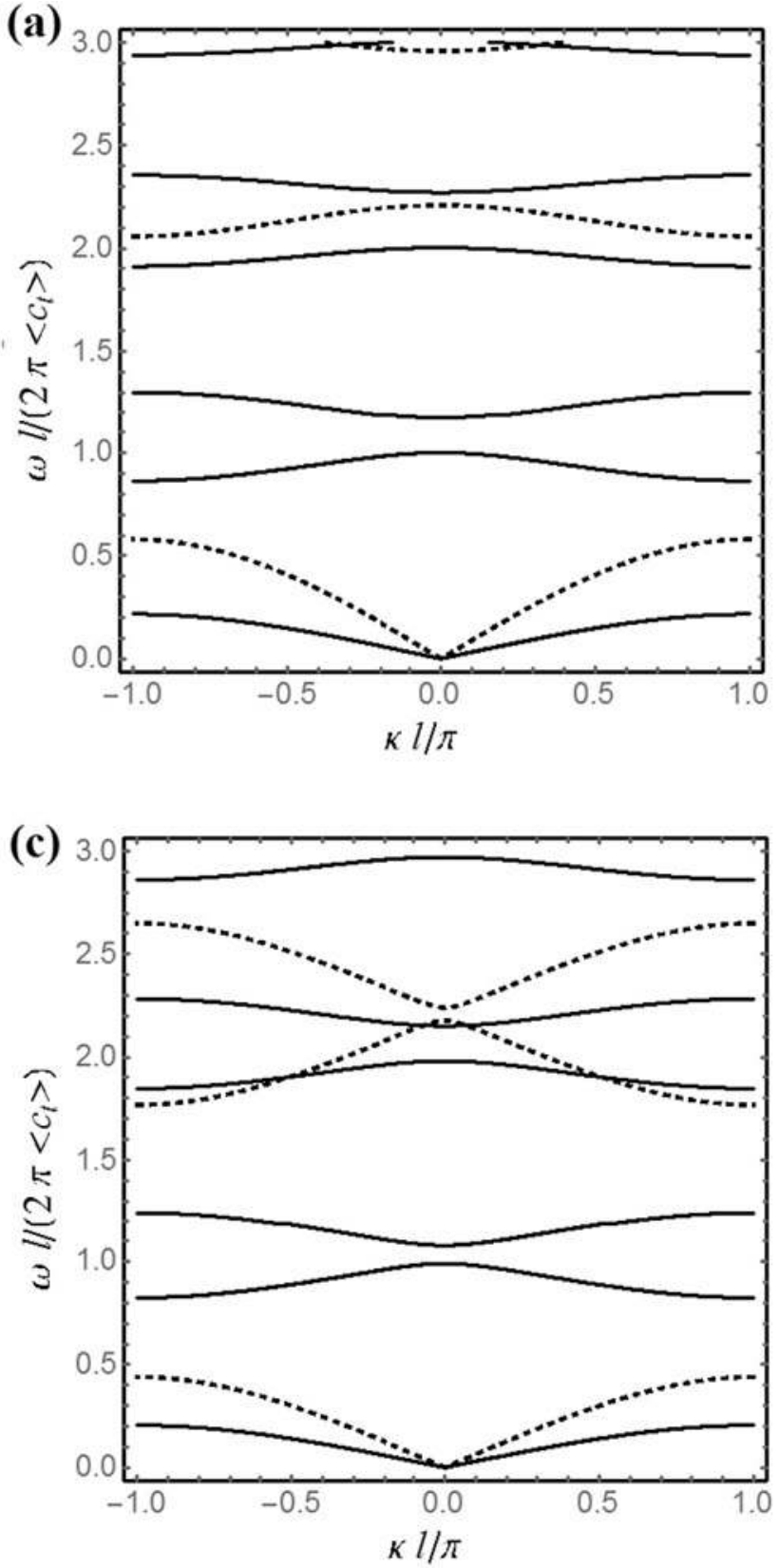

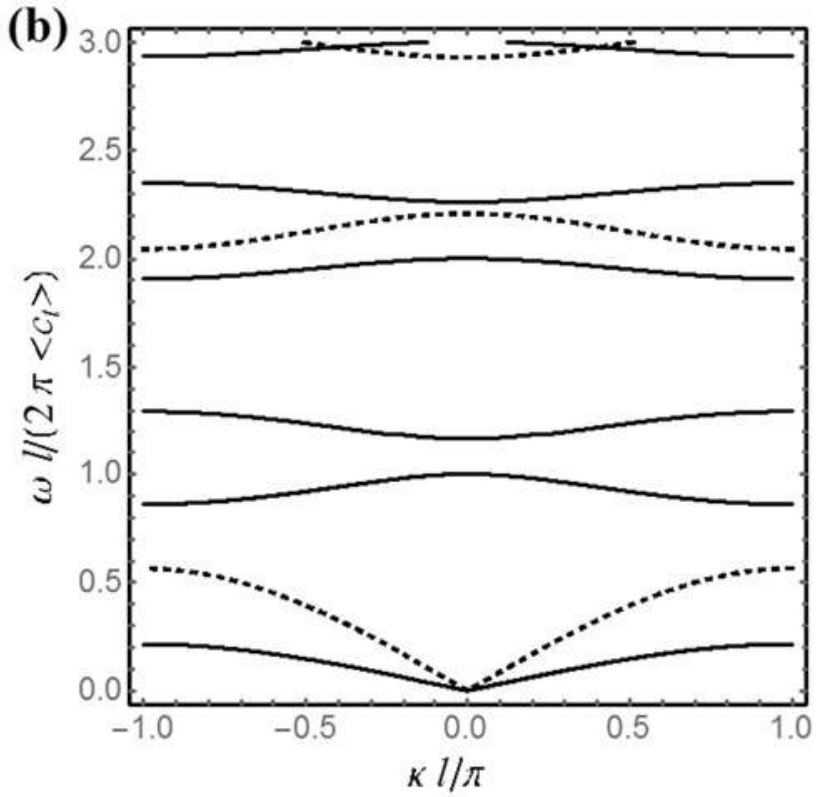

(d)

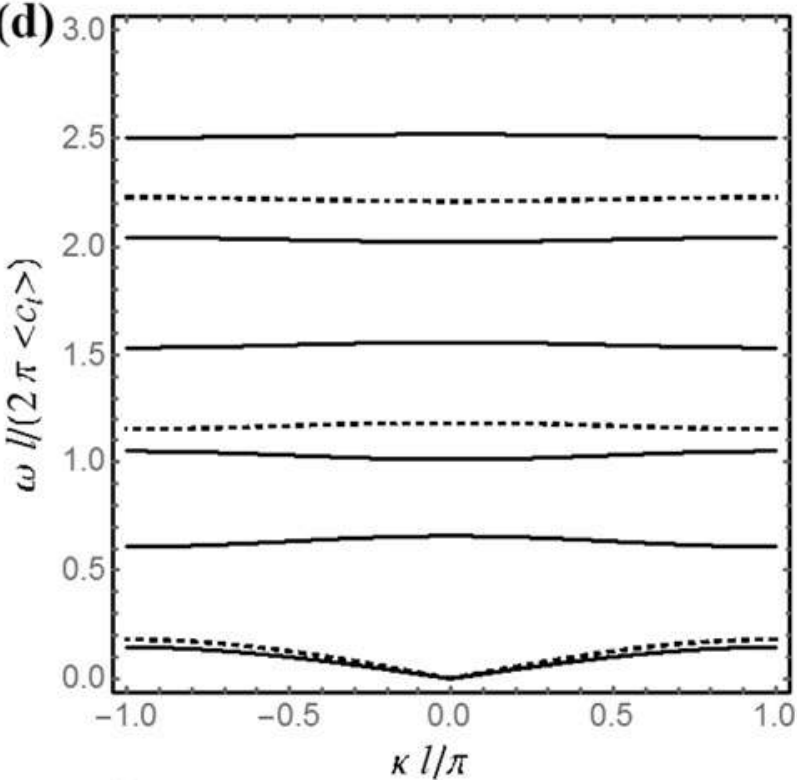

$\mathrm{SH}$, interface

\section{$P$, interface}

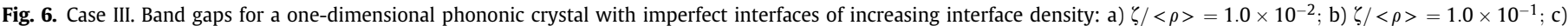
$\zeta /\langle\rho\rangle=1.0$; d) $\zeta /\langle\rho\rangle=1.0 \times 10^{+1}$. The solid lines and the dashed lines represent the dispersion curves of SH and P waves, respectively.

interface model produces the perfect contact conditions when the material is the same in the adherents and in the thin layer, cf. Bövik, 1994, eqns. (35) and (36).

The derivation of our interface law (62)-(63) is based on a different approach, the matched asymptotic expansions. This technique makes use of asymptotic expansions, in particular within the thin layer. The resulting interface law is implicit, nonlocal and explicitly dependent on the elastic properties and mass density of the thin layer (cf. (62)-(63)). Moreover, in view of its implicitly character, the interface law (62)-(63) also depends on the elastic properties and mass density of the adherents. This can be clearly seen in the one-dimensional example considered in Section 5, cf. (81)-(84). Note also that if the same material properties for the thin layer and the adherents are inserted into (83)-(84), then the coefficients $a_{ \pm}$and $b_{ \pm}$vanish and the perfect interface model is reobtained.

In the authors' opinion, the vanishing of the right-hand sides for the same material properties for the thin layer and the adherents can not be considered a general property of an interface law modeling a thin layer. Consider for example the one-dimensional case of a bar made of two different materials, a thin linear elastic adhesive layer of thickness $\varepsilon$ occupying the interval $(-\varepsilon / 2,+\varepsilon / 2)$ 
and two linear elastic adherents occupying the two intervals $(-l / 2-\varepsilon / 2,-\varepsilon / 2)$ and $(+\varepsilon / 2, l / 2+\varepsilon / 2)$. The composite bar is loaded by two opposite forces $F$ at its ends. From the analytical solution of the equilibrium problem, it can be easily seen that the jump $[u]:=u(+\varepsilon / 2)-u(-\varepsilon / 2)$ is equal to $F \varepsilon / E$, with $E$ the elastic modulus of the thin layer. This result is clearly independent of the elastic moduli of the two adherent bars. Actually, in the literature, it has been proved that the jump of the displacement is of order $\varepsilon$ even if adhesive and adherents are made of the same material. This has been rigorously shown by Licht and Michaille via a Gammaconvergence analysis (Licht and Michaille, 1997). Numerical evidence of this fact has been given for example in (Dumont et al., 1999, 2014; Lebon et al., 1997).

Note also that Bövik's interface law Bövik, (1994), eqns. (35) and (36) and the interface law (62)-(63) give similar results when the elastic moduli of the thin layer are much lower than those of the adherents, because they both reduce to the spring-type model. This can be easily seen by assuming the elastic moduli of the thin layer to linearly depend on the thickness $\varepsilon$ and then by neglecting the terms of higher order in $\varepsilon$ in the two interface models. We believe that an original and interesting feature of the interface law (62)-(63) is the possibility of incorporating several classical interface laws, as already described in Section 4.

The effects of the proposed transmission conditions on the band structure of Bloch-Floquet waves propagating in an one dimensional phononic crystal have been discussed on the basis of numerical results. In agreement with (Zheng and Wei, 2009), our results can be interpreted as the equivalence of the binary phononic crystals with imperfect interface and a ternary system in which the third layer has mass, elasticity and a small thickness, as indicated by the results presented in Fig. 3. However, our treatment of a binary phononic crystals with imperfect interface allows to simultaneously consider the effects of the density, stiffness and geometrical parameters on the band structure and it is thus expected to provide theoretical bases for band gap design of ternary or even quaternary or higher (by taking into account different interfacial properties) layered periodic structures.

\section{References}

Bedford, A., 1985. Hamilton's Principle in Continuum Mechanics, Volume 139 of Pitman Research Notes in Mathematics Series. Pitman Advanced Publishing Program.

Benveniste, Y., 2006. An $O\left(h^{N}\right)$ interface model of a three-dimensional curved interphase in conduction phenomena. Proc. R. Soc. A 462, 1593-1617.

Benveniste, Y., Miloh, T., 2001. Imperfect soft and stiff interfaces in two-dimensional elasticity. Mech. Mater 33 (6), 309-323.

Benveniste, Y., 2013. Models of thin interphases and the effective medium approximation in composite media with curvilinearly anisotropic coated inclusions. Int. J. Eng. Sci. 72, 140-154.

Bövik, P., 1994. On the modeling of thin interface layers in elastic and acoustic scattering problems. O. J. Mech. Appl. Meth. 47 (1), 17-42.

Brito-Santana, H., Wang, Y.-S., Rodríguez-Ramos, R., Bravo-Castillero, J., GuinovartDíaz, R., 2015. Dispersive shear-wave propagation in a periodic layered composite with imperfect interfaces. Int. J. Automot. Compos 1 (2-3), 184-204.

Challamel, N., Girhammar, U.A., 2011. Boundary-layer effect in composite beams with interlayer slip. J. Aerosp. Eng. 24 (2), 199-209.

Chen, Y., Li, J., Zhou, J., Tao, H., Zhou, M., Yu, D.Y., 2014. Phononic first band gap of quaternary layered periodic structure with the lumped-mass method. Shock Vib. 2014. Article ID 189539.

Ciarlet, P.G., 1976. Inequalities in Mechanics and Physics, Grundlagen aus den mathematischen Wissenschaften 219. Springer-Verlag, Berlin-Heidelberg-New
York.

Ciarlet, P.G., 1997. Mathematical elasticity. In: Theory of Plates, Series Studies in Mathematics and its Applications, II. North-Holland, Elsevier, Amsterdam.

Dumont, S., Lebon, F., Ould-Khaoua, A., 1999. A numerical tool for periodic het erogeneous media: application to interface in $\mathrm{Al} / \mathrm{SiC}$ composites. ASME. J. Appl. Mech. 67 (1), 214-217.

Dumont, S., Lebon, F., Rizzoni, R., 2014. An asymptotic approach to the adhesion of thin stiff films. Mech. Res. Commun. 58, 24-35.

Ghazaryan, K.B., Piliposyan, D.G., 2011. Interfacial effects for shear waves in one dimensional periodic piezoelectric structure. J. Sound. Vib. 330 (26), 6456-6466.

Hashin, Z., 2002. Thin interphase/imperfect interface in elasticity with application to coated fiber composites. J. Mech. Phys. Solids 50 (12), 2509-2537.

Jensen, J.S., 2003. Phononic band gaps and vibrations in one-and two-dimensional mass-spring structures. J. Sound. Vib. 266 (5), 1053-1078.

Klarbring, A., 1991. Derivation of the adhesively bonded joints by the asymptotic expansion method. Int. J. Eng. Sci. 29, 493-512.

Kushwaha, M.S., Halevi, P., Dobrzynski, L., Djafari-Rouhani, B., 1993. Acoustic band structure of periodic elastic composites. Phy. Rev. Lett. 71 (13), 2022-2025.

Krasuki, F., Lenci, S., 2000. Yield design of bonded joints. Eur. J. Mech. A-Solid 19 (4), 649-667.

Lebon, F., Rizzoni, R., 2010. Asymptotic analysis of a thin interface: the case involving similar rigidity. Int. J. Eng. Sci. 48 (5), 473-486.

Lebon, F., Rizzoni, R., 2011. Asymptotic behavior of a hard thin linear interphase: an energy approach. Int. J. Solids Struct. 48, 441-449.

Lebon, F., Ould-Khaoua, A., Licht, C., 1997. Numerical study of soft adhesively bonded joints in finite elasticity. Comput. Mech. 21, 134-140.

Lebon, F., Zaittouni, F., 2010. Asymptotic modelling of interface taking into account contact conditions: asymptotic expansions and numerical implementation. Int J. Eng. Sci. 48 (2), 111-127.

Lekner, J., 1994. Light in periodically stratified media. J. Acoust. Soc. Amer. A 11, 2892-2899.

Li, Y.-D., Xiong, T., Dong, L., 2015. A new interfacial imperfection coupling mode (IICM) and its effect on the facture behavior of a layered multiferroic composite: anti-plane case. Eur. J. Mech. A-Solid 52, 26-36.

Licht, C., Léger, A., Orankitjaroenb, S., Ould Khaoua, A., 2013. Dynamics of elastic bodies connected by a thin soft viscoelastic layer. J. Math. Pures Appl. 99 (6), 685-703.

Licht, C., Michaille, G., 1997. A modelling of elastic adhesive bonded joints. Adv Math. Sci. Appl. 7 (2), 711-740.

Lions, J.L., Magenes, E., 1968. Problèmes aux limites non homogènes et applications (Dunod, Paris).

Maldovan, M., 2013. Sound and heat revolutions in phononics. Nature 503 (7475), 209-217.

Nairn, J.A., 2007. Numerical implementation of imperfect interfaces. Comp. Mat. Sci. 40, 525-536

Rizzoni, R., Lebon, F., 2012. Asymptotic analysis of an adhesive joint with mismatch strain. Eur. J. Mech. A/Solids 36, 1-8.

Rizzoni, R., Lebon, F., 2013. Imperfect interfaces as asymptotic models of thin curved elastic adhesive interphases. Mech. Res. Commun. 51, 39-50.

Rizzoni, R., Dumont, S., Lebon, F., Sacco, E., 2014. Higher order models for soft and hard elastic interfaces. Int. J. Solids Struct. 51 (23-24), 4137-4148.

Rokhlin, S.I., Wana, Y.J., 1991. Analysis of boundary conditions for elastic wave interaction with an interface between two solids. J. Acoust. Soc. Amer. 89 (2), 503-515.

Rokhlin, S.I., Wang, Y.J., 1992. Equivalent boundary conditions for thin orthotropic layer between two solids: reflection, refraction, and interface waves. J. Acoust. Soc. Amer. 91 (4), 1875-1887.

Seliger, R.L., Whitham, G.B., 1968. Variational principles in continuum mechanics. Proc. Roy. Soc. Lond. Ser. A 305 (1480), 1-25.

Serpilli, M., 2015. Mathematical modeling of weak and strong piezoelectric interfaces. J. Elast. 121 (2), 235-254.

Serpilli, M., Lenci, S., 2016. An overview of different asymptotic models for anisotropic three-layer plates with soft adhesive. Int. J. Solids Struct. 81, 130-140.

Vasseur, J.O., Deymier, P.A., Chenni, B., Djafari-Rouhani, B., Dobrzynski, L., Prevost, D., 2001. Experimental and theoretical evidence for the existence of absolute acoustic band gaps in two-dimensional solid phononic crystals. Phys. Rev. Lett. 86 (14), 3012-3015.

Wu, T.T., Huang, Z.G., Lin, S., 2004. Surface and bulk acoustic waves in twodimensional phononic crystal consisting of materials with general anisotropy. Phys. Rev. B 69 (9), 943011-9430110.

Zheng, M., Wei, P., 2009. Band gaps of elastic waves in 1-D phononic crystals with imperfect interfaces. Int. J. Min. Met. Mater 16 (5), 608-614. 